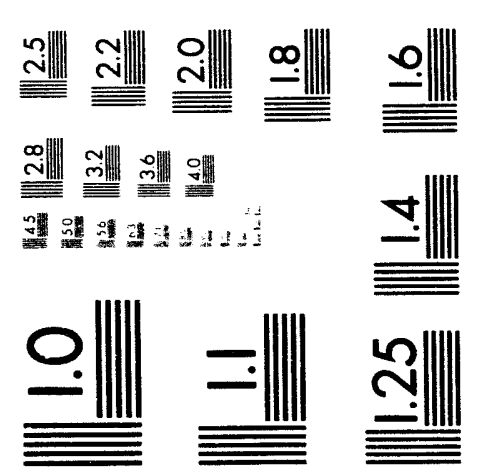



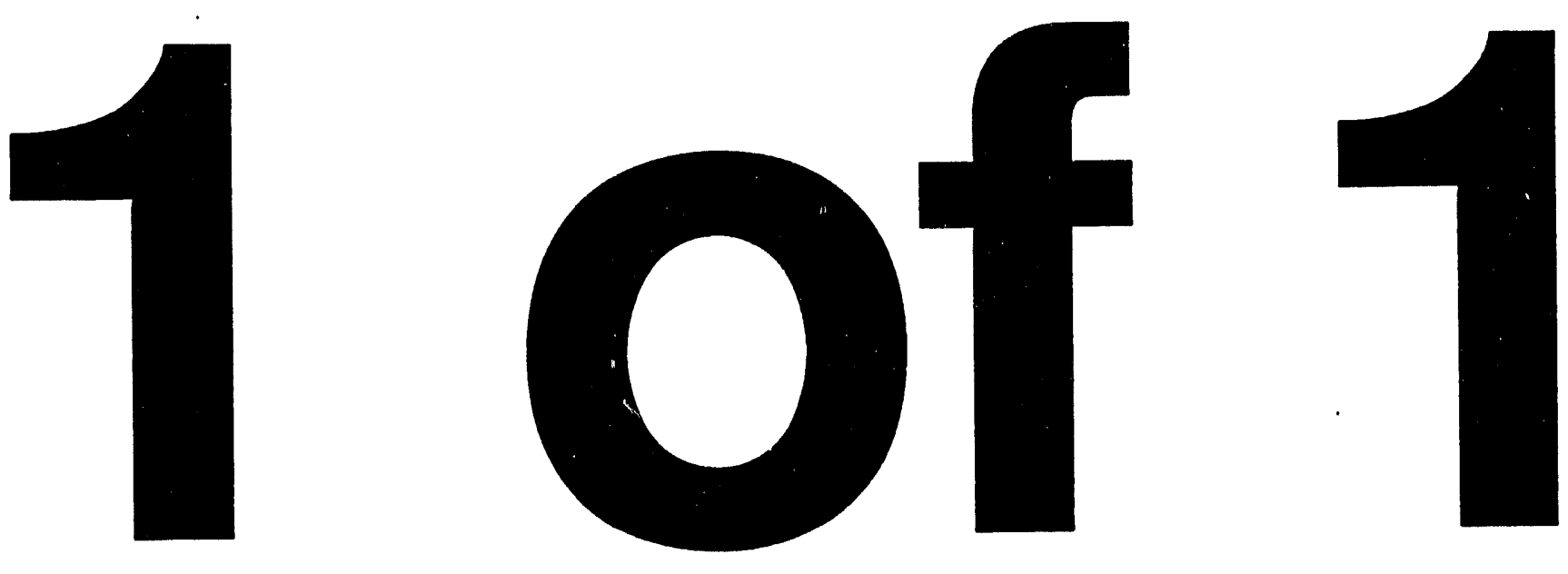
STATISTICAL SUMMARIES OF STREAMFLOW DATA FOR SELECTED GAGING STATIONS ON AND NEAR THE IDAHO NATIONAL ENGINEERING LABORATORY, IDAHO, THROUGH SEPTEMBER 1990

By M.A.J. Stone, Larry J. Mann, and L.C. Kjelstrom

U.S. GEOLOGICAL SURVEY

Water-Resources Investigations Report 92-4196

Prepared in cooperation with U.S. DEPARTMENT OF ENERGY

Boise, Idaho 1993

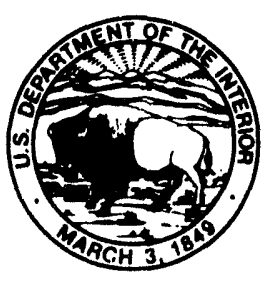




\section{U.S. DEPARTMENT OF THE INTERIOR}

\section{BRUCE BABBITT, Secretary}

\section{U.S. GEOLOGICAL SURVEY}

Dallas L. Peck, Director

For additional information write to:

District Chief

U.S. Geological Survey 230 Collins Road

Boise, ID 83702
Copies of this report can be purchased from:

U.S. Geological Survey

Books and Open-File Reports Section Federal Center, Box 25425

Denver, CO 80225 


\section{CONTENTS}

Page

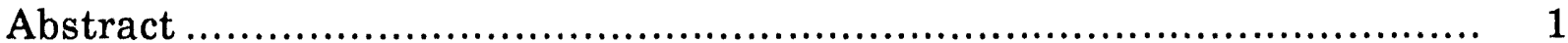

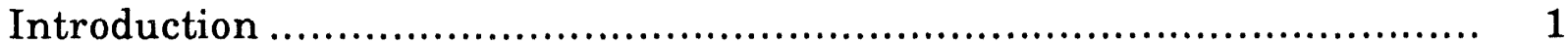

Purpose and scope ........................................................... 3

Previous investigations.................................................... 3

Explanation of statistical summaries.......................................... 5

Monthly and annual flow................................................... 5

Low-flow frequency ........................................................... 5

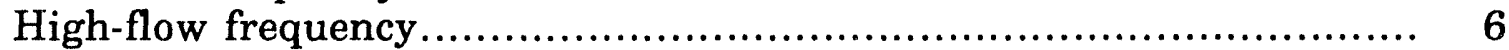

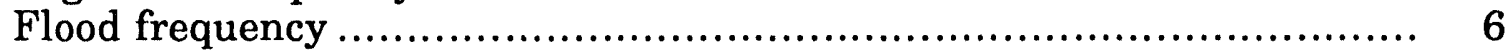

Flow duration...................................................................... 7

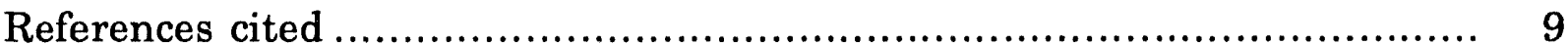

Appendix 1. List of streamflow-gaging stations, in downstream order...... 11

Appendix 2. Descriptions of streamflow-gaging stations and tables of

flow statistics

\section{ILLUSTRATIONS}

Figure 1. Map showing locations of streamflow-gaging stations near the Idaho National Engineering Laboratory

2. Map showing locations of selected facilities and streamflowgaging stations at the Idaho National Engineering Laboratory....

3-5. Graphs showing:

3. Annual mean discharge frequency for a selected streamflow-gaging station...

4. Maximum-median-minimum discharge for a selected streamflow-gaging station ............................... 8

5. Annual mean and mean annual discharge for a selected streamflow-gaging station 


\section{CONVERSION FACTORS, VERTICAL DATUM, AND ABBREVIATIONS}

\section{Multiply}

acre

cubic foot per second $\left(\mathrm{ft}^{3 / \mathrm{s}}\right)$

foot $(\mathrm{ft})$

mile (mi)

square mile $\left(\mathrm{mi}^{2}\right)$
By

4,047

0.02832

0.3048

1.609

2.590
Toobtain

square meter

cubic meter per second

meter

kilometer

square kilometer

Sea level: In this report, "sea level" refers to the National Geodetic Vertical Datum of 1929 - a geodetic datum derived from a general adjustment of the firstorder level nets of the United States and Canada, formerly called Sea Level Datum of 1929.

Abbreviations: ID, Idaho

INEL, Idaho National Engineering Laboratory

$\mathrm{N}$-day, number of consecutive days within a 1-year period

USGS, U.S. Geological Survey

WSP, Water-Supply Paper

Note: Because of the inconsistent nomenclature in the various computer programs used to generate this report, the terms "flow" and "discharge" are used interchangeably. 


\title{
STATISTICAL SUMMARIES OF STREAMFLOW DATA FOR SELECTED GAGING STATIONS ON AND NEAR THE IDAHO NATIONAL ENGINEERING LABORATORY, IDAHO, THROUGH SEPTEMBER 1990
}

\author{
By \\ M.A.J. Stone, Larry J. Mann, and L.C. Kjelstrom
}

\begin{abstract}
Statistical summaries and graphs of streamflow data were prepared for 13 gaging stations with 5 or more years of continuous record on and near the Idaho National Engineering Laboratory. Statistical summaries of streamflow data for the Big and Little Lost Rivers and Birch Creek were analyzed as a requisite for a comprehensive evaluation of the potential for flooding of facilities at the Idaho National Engineering Laboratory.

The type of statistical analyses performed depended on the length of streamflow record for a gaging station. Streamflow statistics generated for stations with 5 to 9 years of record were: (1) magnitudes of monthly and annual flows; (2) duration of daily mean flows; and (3) maximum, median, and minimum daily mean flows. Streamflow statistics generated for stations with 10 or more years of record were: (1) magnitudes of monthly and annual flows; (2) magnitudes and frequencies of daily low, high, instantaneous peak (flood frequency), and annual mean flows; (3) duration of daily mean flows; (4) exceedance probabilities of annual low, high, instantaneous peak, and mean annual flows; (5) maximum, median, and minimum daily mean flows; and (6) annual mean and mean annual flows.
\end{abstract}

\section{INTRODUCTION}

The Idaho National Engineering Laboratory (INEL) includes about $890 \mathrm{mi}^{2}$ of the eastern Snake River Plain in southeastern Idaho (fig. 1). The Big Lost River is the principal stream that flows onto and terminates on the INEL. The Little Lost River and Birch Creek drain intermontane valleys west and north of the INEL and terminate in the northern part. The Big and Little Lost Rivers and Birch Creek terminate in a series of playas or sinks on and adjacent to the INEL, as shown in figure 1 .

Streamflow records have been collected at many gaging stations on the Big and Little Lost Rivers and Birch Creek. Statistical data based on the streamflow records provide information about the areal and spatial quantity of water. This information is useful for studies of streamflow seepage losses, ground-water recharge, and flood-control analysis. 


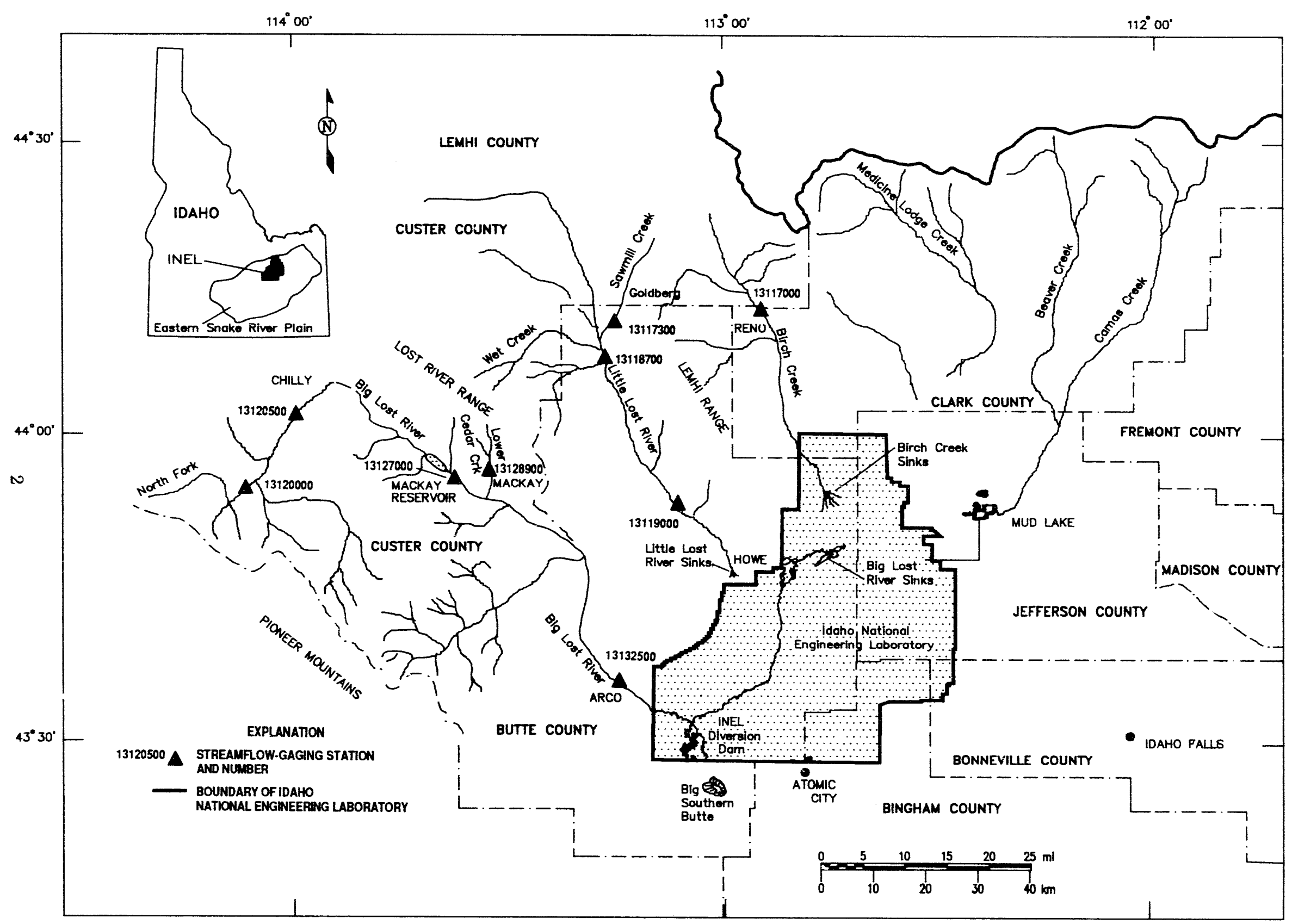

Figure 1.--Locations of streamflow-gaging stations near the Idaho National Engineering Laboratory. 
The need for flood control at the INEL was recognized in the early 1950's when the Test Reactors Area and the Idaho Chemical Processing Plant were threatened by flooding in the Big Lost River. A small diversion dam was constructed across the Big Lost River in 1958 to divert water from the river through a diversion channel into a spreading area (fig. 2). Repeated threats of flooding in the late 1960's, early 1970's, and early 1980's occurred when the Big Lost River filled Playas 1 and 2 and overflowed into Playa 3 near an INEL facility (fig. 2). Discharges of a few hundred cubic feet per second and air temperatures as cold as $-47^{\circ} \mathrm{F}\left(-44^{\circ} \mathrm{C}\right)$ during the winter of $1983-84$ caused ice jams that imposed a danger of localized flooding. The diversion channel was enlarged in 1984 to provide additional flood control; the dam across the Big Lost River and the containment dikes along the diversion channel and spreading areas were raised several feet. The capacity of the diversion channel and two low swales near the channel is $9,300 \mathrm{ft}^{3} / \mathrm{s}$ (Bennett, 1986).

Statistical summaries of streamflow data were prepared for gaging stations on the Big and Little Lost Rivers and Birch Creek in cooperation with the U.S. Department of Energy.

\section{Purpose and Scope}

This report presents statistical summaries of streamflow data for 13 gaging stations with 5 or more years of continuous record. Records through September 30,1990 , or September 30 of the last year of record, if gaging stations were discontinued prior to 1990 , were used in computing monthly and mean annual flows, high-flow frequency, and flow-duration data. Low-flow frequency data were computed on the basis of the 1991 climatic year, which ends March 31, 1991. Flood-frequency data were computed using records through the 1990 water year (ending September 30, 1990).

For the four gaging stations with 5 to 9 years of continuous record, the following information is provided: (1) magnitudes of monthly and annual flows; (2) duration of daily mean flows; and (3) maximum, median, and minimum daily mean flows. For the nine gaging stations with 10 or more years of record, the following information is provided: (1) magnitudes of monthly and annual flows; (2) magnitudes and frequencies of daily low, high, instantaneous peak (flood frequency), and annual mean flows; (3) duration of daily mean flows; (4) exceedance probabilities of annual low, high, instantaneous peak, and mean annual flows; (5) maximum, median, and minimum daily mean flows; and (6) annual mean and mean annual flows.

\section{Previous Investigations}

Several investigations have been conducted to evaluate flow characteristics of streams at the INEL, particularly the Big Lost River and INEL diversion. Lamke (1969) described stage-discharge relations for the Big Lost River. Carrigan (1972) described the probability of a flood exceeding the capacity of the INEL 


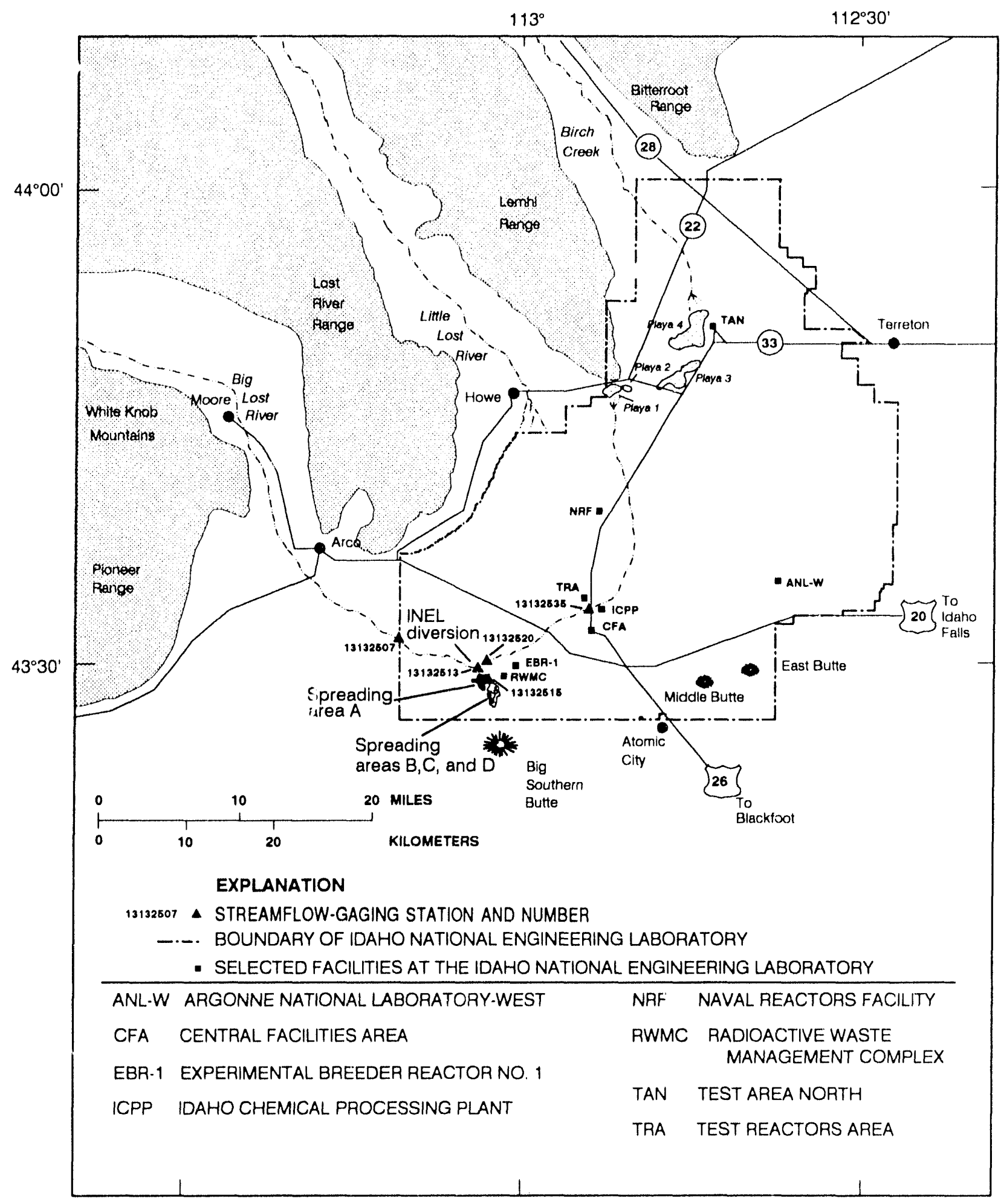

Figure 2,--Locations of selected facilities and streamflow-gaging stations at the Idaho National Engineering Laboratory. 
diversion. The capacity of the INEL diversion channel was evaluated by Bennett (1986), who also described streamflow losses along the Big Lost River at the INEL (1990). Koslow and Vanhaaften (1986) described the area that would be inundated by the probable maximum flood on the Big Lost River.

\section{EXPLANATION OF STATISTICAL SUMMARIES}

Statistical summaries of streamflow data are presented (back of report) for 13 gaging stations shown on figures 1 and 2 . The description of each gaging station includes the location of the gage, drainage area, period of record, description of the gage, remarks pertinent to the quality of streamflow records and to conditions that affect the flow, and extremes for the period of record.

Water is diverted upstream from many gaging stations. The amount of water diverted and the time of diversion are variable, depending on the demand and availability of water. The Big Lost River is regulated by Mackay Reservoir. The statistical summaries are based on recorded streamflow at the gaging station.

Tabulations for each gaging station (back of report) include the magnitudes of monthly and annual flows, magnitudes and frequencies of annual low and high flows, magnitudes and frequencies of instantaneous peak flows, and duration of daily mean flows. Monthly and annual data were processed using USGS computer program W4422 (Price and Meeks, 1977); low-flow, high-flow, and flow-duration data were processed using USGS computer program A969 (Dempster, 1984); and flood-frequency data were processed using USGS computer program J407 (Kirby, 1981).

\section{Monthly and Annual Flow}

The monthly and annual flow tabulations for the period of record include the maximum, minimum, and mean monthly and mean annual flow, the standard deviation of the means, the coefficient of variation (ratio of the standard deviation to the mean), and the percentage of average annual runoff for each month.

\section{Low-Flow Frequency}

The low-flow tabulations show the data necessary to plot standard low-flow frequency curves, which are based on the log-Pearson Type III frequency distribution. The tabulations show annual minimum mean flows for periods of 1 , $3,7,14,30,60,90,120$, and 183 consecutive days for recurrence intervals of $2,5,10$, 20,50 , and 100 years; the associated annual nonexceedance probabilities are 50, $20,10,5,2$, and 1 percent, respectively. The annual minimum mean flows are based on the climatic year. Recurrence intervals for low flows represent the average length of time between occurrences of annual minimum mean flows that 
are less than the stated flow magnitude. Nonexceedance probability is the probability or chance, expressed as a percentage, that the annual minimum mean flow will be less than the stated magnitude in any given year. Recurrence intervals generally were reported only to twice the period of record. Tahulations based on records of more than 40 years were extended to the 100-year (1-percent) interval. If the period of record is not longer than one-half of the minimurn reported recurrence interval for frequency calculations, the computer program FLOWSTAT (Rogers and Werley, 1992) automatically flags (\#) the frequency column to indicate that the values shown may be unreliable.

\section{High-Flow Frequency}

The high-flow frequency tabulations show the data necessary to plot standard high-flow frequency curves, which are based on the log-Pearson Type III frequency distribution. The tabulations show the annual maximum mean flows for periods of $1,3,7,15,30,60$, and 90 consecutiva days for recurrence intervals of $2,5,10,25,50$, and 100 years; the associated annual exceedance probabilities are $50,20,10,4,2$, and 1 percent, respectively. The annual maximum mean flows are based on the water year. Recurrence intervals for high flows represent the average length of time between occurrences of annual maximum mean flows equal to or greater than the stated flow magnitude. Exceedance probability is the probability or chance, expressed as a percentage, that the annual maximum mean flow will equal or exceed the stated magnitude in any given year. The criteria for extending frequency curves for high-flow data were the same as for the low-flow data. If the period of record did not meet USGS criteria for frequency calculations, the frequency column is flagged (\#) to indicate that the values shown may be unreliable.

\section{Flood Frequency}

The flood-frequency tabulations show the data necessary to plot standard flood-frequency curves, which are based on log-Pearson Type III frequency distribution. These data are magnitudes of instantaneous peak flows at selected recurrence intervals (annual exceedance probabilities). The log-Pearson Type III frequency distribution was fitted to recorded data for stations with 10 or more years of record using procedures recommended by the U.S. Advisory Committee on Water Data (1981). A log-Pearson Type III frequency distribution incorporates three factors-mean, standard deviation, and skew coefficient-which affect position, slope, and curvature of the distribution graph, respectively. Generalized skew coefficients are weighted with gaging station skew coefficients (determined from peak flow analysis) to obtain a more reliable flood-frequency distribution curve than could be obtained using either generalized or station skew coefficients. Generalized skew coefficients were determined by reference to a report by Kjelstrom and Moffatt (1981). 
The flood-frequency tabulations list the magnitudes of annual instantaneous peak flows for recurrence intervals of $2,5,10,25,50$, and 100 years; the associated annual exceedance probabilities are $50,20,10,4,2$, and 1 percent, respectively.

\section{Flow Duration}

The flow-duration tabulations show the data necessary to plot a standard flow-duration curve, which is a cumulative frequency curve that shows the percentage of time that specified daily flows were equaled or exceeded during the period of record. The tabulations show the flows, in cubic feet per second, that were equaled or exceeded for a given percentage of time.

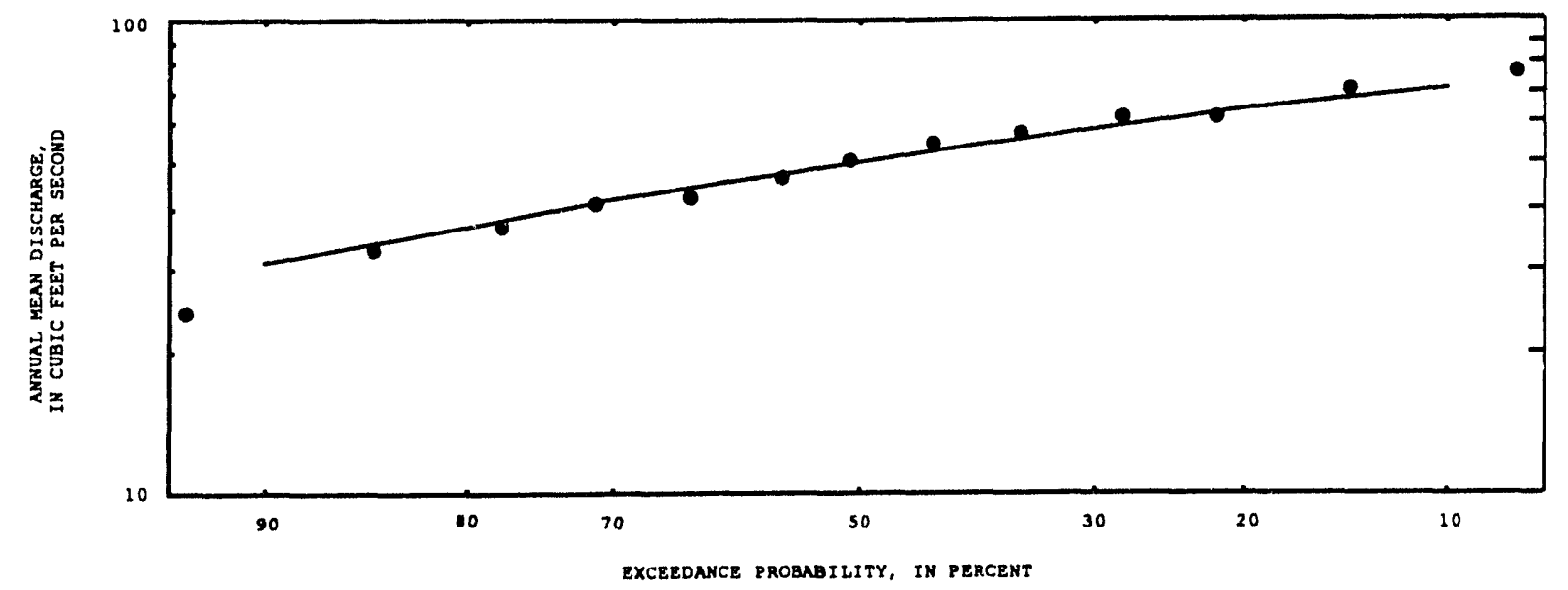

Figure 3.--Annual mean discharge frequency for a selected streamflow-gaging station.

The annual mean discharge frequency graph (fig. 3) shows the relation between annual mean flows and their associated exceedance probabilities at a specified gaging station. The annual mean flows were calculated from a $365 \mathrm{~N}$ day analysis of daily values using ANNIE, a USGS hydrologic analysis and data management program (Lumb and others, 1990). The resulting values were fitted with a log-Pearson Type III frequency distribution to derive the exceedance probabilities. 


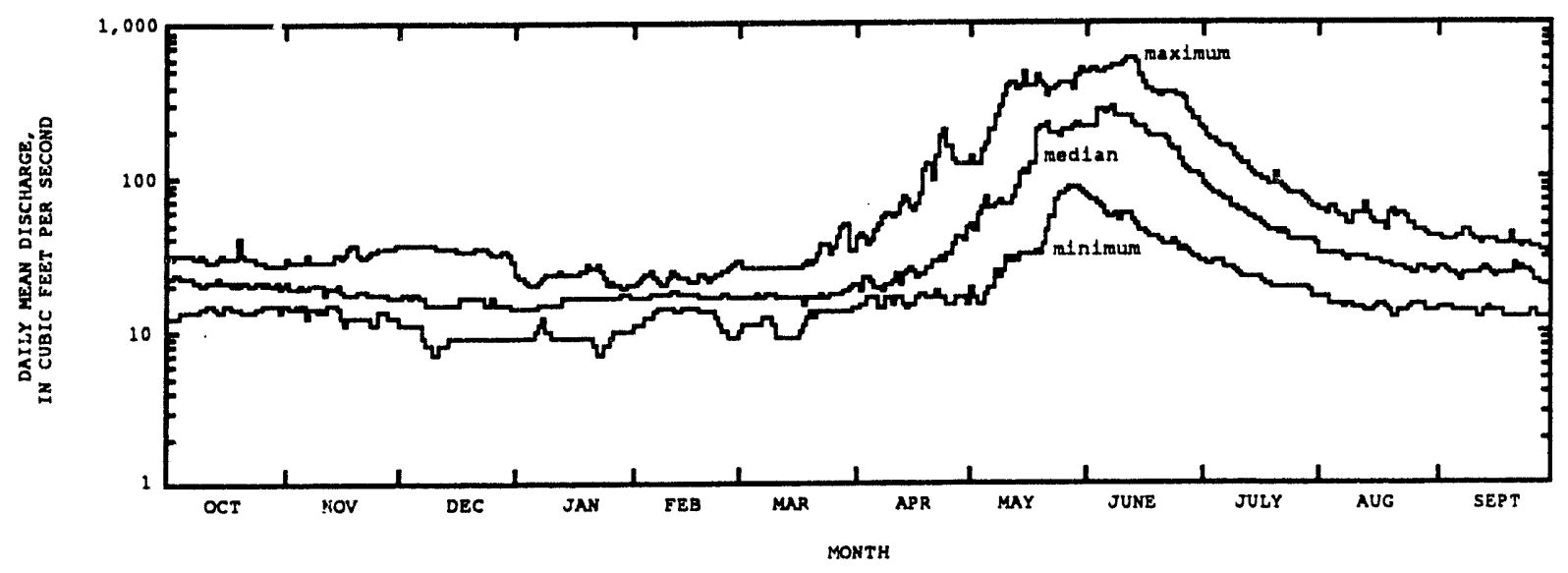

Figure 4.--Maximum-median-minimum discharge for a selected streamflow-gaging station.

The maximum-median-minimum discharge graph (fig. 4) shows the highest, median, and lowest flow past the gaging station during the period of record for each day of the year. A minimum of $1 \mathrm{ft}^{3} / \mathrm{s}$, or four logarithmic cycles below the maximum flow, was the minimum discharge graphed. Maximum, median, and minimum flows were computed using WATSTORE program K609 (Bergmann, 1981) and were plotted using ANNIE.

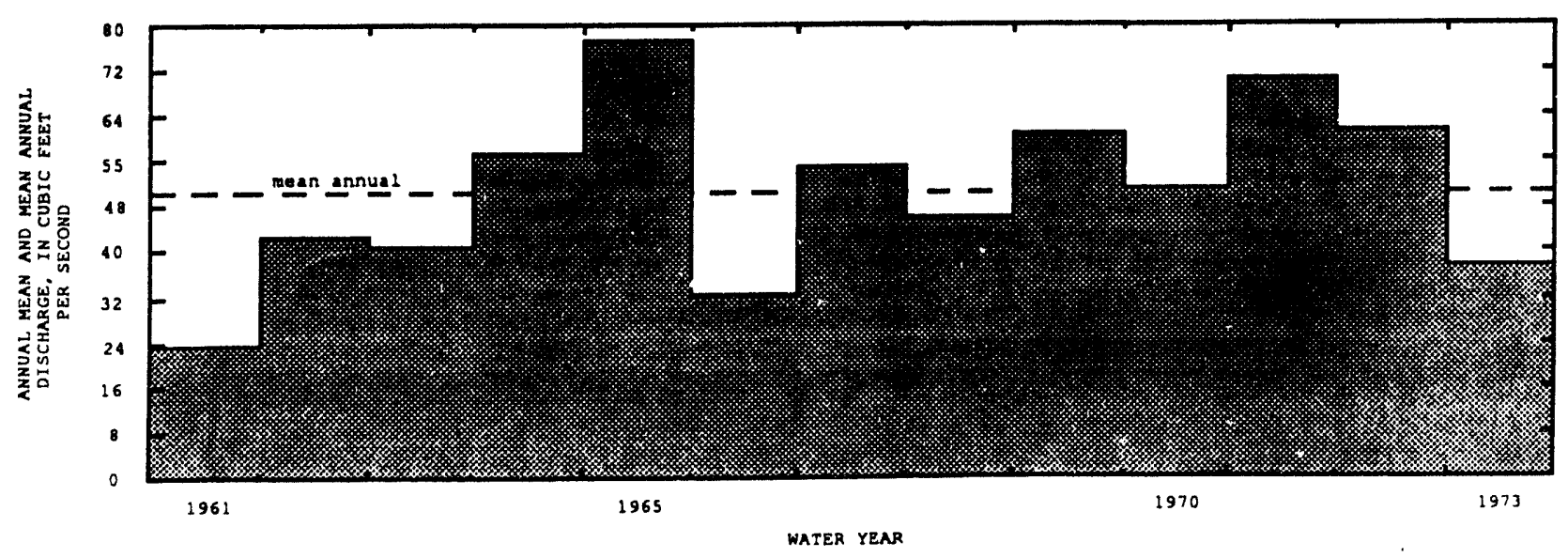

Figure 5.--Annual mean and mean annual discharge for a selected streamflow-gaging station.

The annual mean and mean annual discharge graph (fig. 5) shows how annual mean flow differs from the long-term mean annual flow for the gaging station. All values were computed and plotted using ANNIE. The annual mean is displayed with a solid line and the mean annual with a dashed line. For periods when partial records or no records were available, only the mean annual is shown. 


\section{REFERENCES CITED}

Bennett, C.M., 1986, Capacity of the diversion channel below the flood-control dam on the Big Lost River at the Idaho National Engineering Laboratory: U.S. Geological Survey Water-Resources Investigations Report 86-4204, 25 p.

Bennett, C.M., 1990, Streamflow losses and ground-water level changes along the Big Lost River at the Idaho National Engineering Laboratory, Idaho: U.S. Geological Survey Water-Resources Investigations Report 90-4067, 49 p.

Bergmann, J.M., 1981, Daily values summary statistics (Program K609), chap. IV, sec. K of Hutchison, N.E., compiler, WATSTORE user's guide: U.S. Geological Survey Open-File Report 75-426, v. 1, p. K-1 to K-17.

Carrigan, P.H., Jr., 1972, Probability of exceeding capacity of the flood-control system at the National Reactor Testing Station, Idaho: U.S. Geological Survey Open-File Report IDO-22052, 102 p.

Dempster, G.R., Jr., 1984, Meek's daily values statistics (Programs A969 and A193), chap. IV, sec. G, of Hutchison, N.E., compiler, WATSTORE user's guide: U.S. Geological Survey Open-File Report 75-426, v. 1, p. G-1 to G-38.

Kirby, William, 1981, Annual flood frequency analysis using U.S. Water Resources Council guidelines (Program J407), chap. I, sec. C, of Hutchison, N.E., compiler, WATSTORE user's guide: U.S. Geological Survey Open-File Report 76-435, v. 4, p. C-1 to C-57.

Kjelstrom, L.C., and Moffatt, R.L., 1981, A method of estimating flood-frequency parameters for streams in Idaho: U.S. Geological Survey Open-File Report 81-909, 99 p.

Koslow, K.N., and Vanhaaften, D.H., 1986, Flood routing analysis for a failure of Mackay Dam: EG\&G Idaho, Inc., report no. EGG-EP-7184, 33 p., 3 apps.

Lamke, R.D., 1969, Stage-discharge relations on Big Lost River within National Reactor Testing Station, Idaho: U.S. Geological Survey Open-File Report IDO-22050, 29 p.

Lumb, A.M., Kittle, J.L., Jr., and Flynn, K.M., 1990, Users manual for ANNIE, a computer program for interactive hydrologic analyses and data management: U.S. Geological Survey Water-Resources Investigations Report 89-4080, 236 p.

Price, W.E., Jr., and Meeks, W.C., 1977, Daily values of monthly and annual statistics (Program W4422), chap. IV, sec. F, of Hutchison, N.E., compiler, WATSTORE user's guide: U.S. Geological Survey Open-File Report 74-426, v. 1, p. F-1 to F-46. 
Rogers, G.D., and Werley, M.R., 1992, A computer program (FLOWSTAT) for summarizing daily and peak streamflow statistics: U.S. Geological Survey Open-File Report 92-115, $17 \mathrm{p}$.

U.S. Water Resources Ccuncil, 1981, Guidelines for determining flood flow frequency [revised]: Washington, D.C., Hydrology Committee Bulletin 17B, 28 p., 14 apps. 


\section{Appendix 1}

\section{LIST OF STREAMFLOW-GAGING STATIONS, IN DOWNSTREAM ORDER}

Gaging station

number

13117000

13117300

13118700

13119000

13120000

13120500

13127000

13128900

13132500

13132513

13132515

13132520

13132535
Gaging station name

Page

Birch Creek near Reno, ID.......................................... 14

Sawmill Creek near Goldberg, ID .................................. 16

Little Lost River below Wet Creek, near Howe, ID ................. 18

Little Lost River near Howe, ID......................................... 20

North Fork Big Lost River at Wild Horse, near Chilly, ID........... 22

Big Lost River at Howell Ranch, near Chilly, ID .................... 24

Big Lost River below Mackay Reservoir, near Mackay, ID......... 26

Lower Cedar Creek above diversion 3, near Mackay, ID.............. 28

Big Lost River near Arco, ID ............................................ 30

INEL diversion at head near Arco, ID ............................. 32

INEL diversion at outlet of spreading area A near Arco, ID ....... 33

Big Lost River below INEL diversion near Arco, ID ................. 34

Big Lost River at Lincoln Boulevard Bridge near Atomic City, ID 35 


\section{Appendix 2}

DESCRIPTIONS OF STREANFLOW-GAGING STATIONS AND TABLES OF FLOW STATISTICS 
MUD LAKE-LOST RIVEK BASIN

13117000 BIRCH CREEK NEAR RENO, ID

LOCATION.--Lat $44^{\circ} 12^{\prime}$, long $112^{\circ} \mathrm{5} 7^{\prime}$, in gec.13, T.10 N., R.29 E., Clark County, Hydrologlc Unlt 17040216 , on left bank 200 ft west of State Highway 28, $2 \mathrm{ml}$ south of the Lemhl-Clark County 11ne, $5 \mathrm{ml}$ southeast of former Reno Post of $1 \mathrm{ce}$, and $35 \mathrm{ml}$ west of Dubols.

DRAINAGE AREA. $-320 \mathrm{ml}$; approximately.

PERIOD OF RECORD.--September 1910 to June 1912 (publ1shed as "near Kaufman"), Apr11 1921 to January 1923 , October 1950 to September 1963.

GAGE.--Water-stage recorder. Altitude of gage $1 \mathrm{~s} 6,240 \mathrm{ft}$ above sea level (by barometer). Prlor to oct. 1, 1950 , nonrecording gage at site $0.5 \mathrm{mi}$ downstream at different datum.

REMARKS, --Small diversions for stock ranches and hay meadows above station.

EXTREMES FOR PERIOD OF RECORD.--Maximum discharge, $220 \mathrm{ft}$ \%/s Apr. 1, 1962 (gage helght, $2.19 \mathrm{ft}$ ); max1mum gage helght observed, 3.11 ft Jan. 25, 1962 (backwater from 1ce): minimum dally mean discharge, $50 \mathrm{ft} / \mathrm{s}$, Jan. 12, 1963; minimum gage helght, $1.33 \mathrm{ft}$, Jan. 23, 1963 .

Summary of monthly and annual discharges, 1911, 1922, 1951-63

\begin{tabular}{|c|c|c|c|c|c|c|}
\hline Month & $\begin{array}{l}\text { Maximum } \\
\text { (ft } 3 / s)\end{array}$ & $\begin{array}{l}\text { Minimum } \\
\left(\mathrm{ft}^{3} / \mathrm{s}\right)\end{array}$ & $\begin{array}{c}\text { Mean } \\
\left(\mathrm{ft}^{3} / \mathrm{s}\right)\end{array}$ & $\begin{array}{l}\text { stan- } \\
\text { dard } \\
\text { devia- } \\
\text { tlon } \\
\left(f^{\prime} / g\right)\end{array}$ & $\begin{array}{l}\text { Coefti- } \\
\text { clent of } \\
\text { vari- } \\
\text { ation }\end{array}$ & $\begin{array}{l}\text { Per- } \\
\text { centage } \\
\text { of } \\
\text { annual } \\
\text { runoff }\end{array}$ \\
\hline October & 94 & 73 & 79 & 6.2 & 0.08 & 8.3 \\
\hline November & $8 \theta$ & 68 & 78 & 5.1 & 0.06 & 8.3 \\
\hline December & 88 & 67 & 78 & 4.8 & 0.06 & 8.3 \\
\hline January & 93 & 63 & 78 & 6.5 & 0.08 & 8.3 \\
\hline February & 90 & 69 & 79 & 5.4 & 0.07 & 8.3 \\
\hline March & 92 & 72 & 79 & 5.8 & 0.07 & 8.4 \\
\hline Apr11 & 89 & 73 & 81 & 5.1 & 0.06 & 8.6 \\
\hline May & 90 & 74 & 81 & 4.5 & 0.06 & 8.6 \\
\hline June & 89 & 73 & 79 & 4.8 & 0.06 & 8.4 \\
\hline July & 83 & 68 & 76 & 3.9 & 0.05 & 8.1 \\
\hline August & 86 & 70 & 77 & 5.3 & 0.07 & 8.1 \\
\hline September & 87 & 72 & 78 & 5.9 & 0.08 & 8. 3 \\
\hline Annual & 89 & 70 & 79 & 4.5 & 0.06 & 100 \\
\hline
\end{tabular}
based on pertod of record 1911, 1922, 1951-63

Dlacharge in et'/s, for Indlcated recurrence lnterval, In yeara, and exceedance probability, in percent

\begin{tabular}{|c|c|c|c|c|c|}
\hline $\begin{array}{c}2 \\
50\end{array}$ & $\begin{array}{r}5 \\
20\end{array}$ & $\begin{array}{r}10 \\
10\end{array}$ & $\begin{array}{l}25 \\
48\end{array}$ & $\begin{array}{l}50 \\
21\end{array}$ & $\begin{array}{r}100 \\
1\end{array}$ \\
\hline 95 & 113 & 134 & 171 & 209 & 257 \\
\hline
\end{tabular}

Duration table of dally mean flow for period of record 1961-73

Magnitude and frequency of annual low flow, based on perlod of record 1912, 1922, 1952-63

\begin{tabular}{|c|c|c|c|c|c|c|}
\hline \multirow{3}{*}{$\begin{array}{l}\text { Perlod } \\
\text { (con- } \\
\text { secu- } \\
\text { tive } \\
\text { days) }\end{array}$} & \multicolumn{6}{|c|}{ 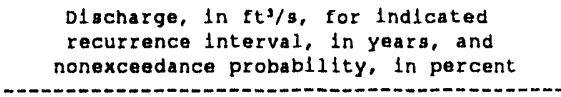 } \\
\hline & 2 & 5 & 10 & 20 & 504 & 100 \\
\hline & 50 & 20 & 104 & 53 & 24 & 17 \\
\hline 1 & 72 & 64 & 59 & 55 & 50 & 46 \\
\hline 3 & 72 & 65 & 61 & 57 & 52 & 48 \\
\hline 7 & 72 & 67 & 63 & 60 & 56 & 53 \\
\hline 14 & 73 & 68 & 65 & 62. & 58 & 56 \\
\hline 30 & 73 & 69 & 67 & 65 & 64 & 62 \\
\hline 60 & 74 & 71 & 69 & 67 & 65 & 64 \\
\hline 90 & 75 & 72 & 70 & 68 & 66 & 65 \\
\hline 120 & 76 & 72 & 70 & 68 & 66 & 65 \\
\hline 183 & 77 & 73 & 71 & 70 & 68 & 67 \\
\hline
\end{tabular}

Magnitude and frequency of annual high flow, based on pertod of record 1911, 1922, 1951-63

\begin{tabular}{|c|c|c|c|c|c|c|}
\hline \multirow{2}{*}{$\begin{array}{l}\text { Period } \\
\text { lcon- } \\
\text { encu- } \\
\text { tive }\end{array}$} & \multicolumn{6}{|c|}{$\begin{array}{l}\text { D1scharge, In } \mathrm{ft}^{3 / \mathrm{s}} \text {, for Indicated } \\
\text { recurrence Interval, in years, and } \\
\text { exceedance probability, in percent }\end{array}$} \\
\hline & 2 & 5 & 10 & 25 & 50 & 100 \\
\hline daysi) & 50. & 204 & 104 & 44 & 24 & 11 \\
\hline 1 & 88 & 98 & 107 & 120 & 131 & 142 \\
\hline 3 & 87 & 96 & 103 & 114 & 123 & 132 \\
\hline 7 & 86 & 94 & 99 & 105 & 110 & 115 \\
\hline 15 & 85 & 91 & 95 & 100 & 103 & 106 \\
\hline 30 & 84 & 89 & 91 & 94 & 96 & 97 \\
\hline 60 & 82 & 87 & 89 & 92. & 93 & 95 \\
\hline 90 & 81 & 86 & 88 & 91 & 93 & 95 \\
\hline
\end{tabular}

\begin{tabular}{|c|c|c|c|c|c|c|c|c|c|c|c|c|c|c|c|c|}
\hline 14 & 51 & 10 & 151 & 204 & 301 & 40 & 501 & 604 & 704 & 801 & 901 & 954 & 981 & 994 & 99.5 & 99.98 \\
\hline 94 & 89 & 87 & 85 & 54 & 82 & 80 & 79 & 77 & 76 & 75 & 73 & 71 & 68 & 67 & 66 & 57 \\
\hline
\end{tabular}

- Length of record used in calculation may yleld unrellable values for this column. 

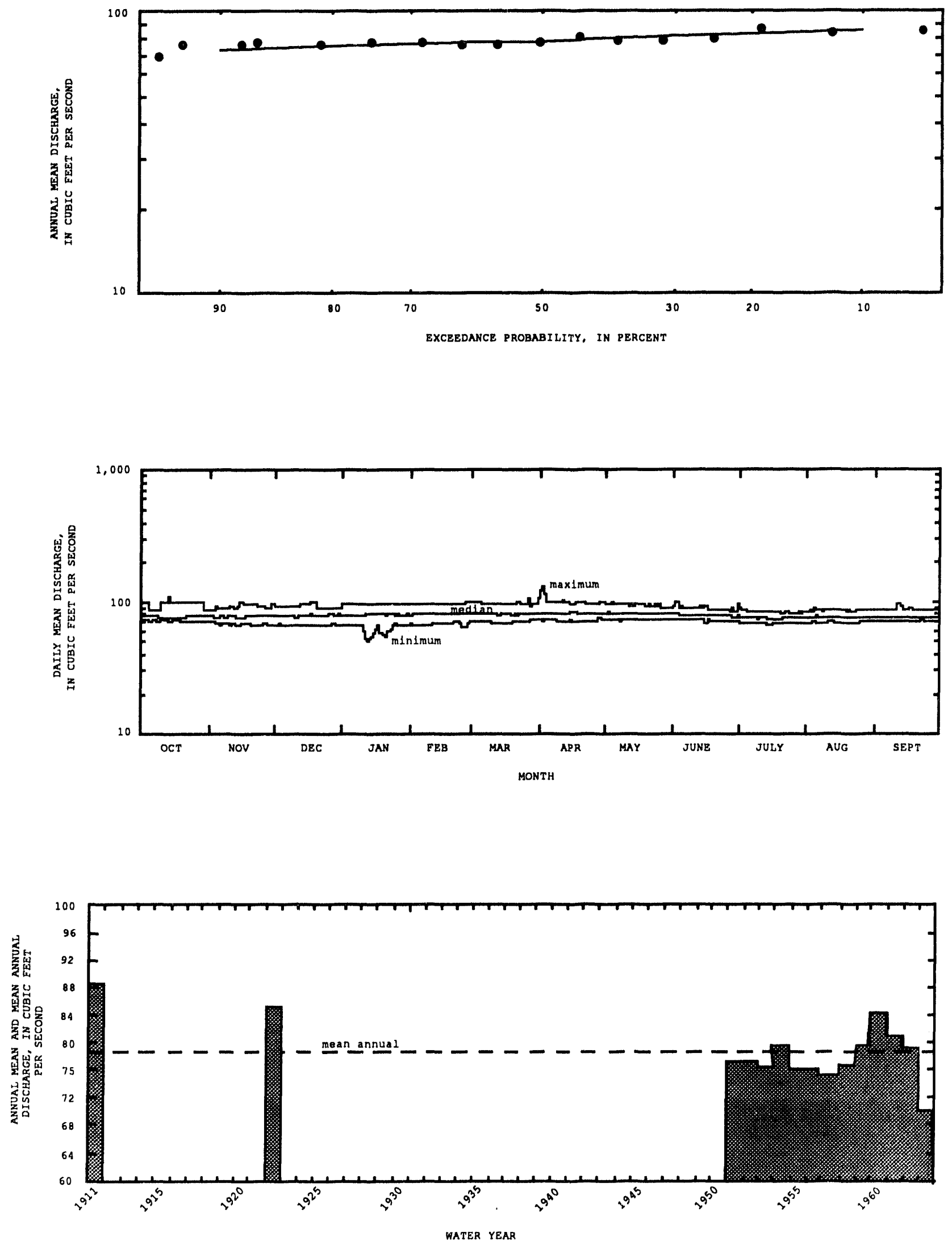


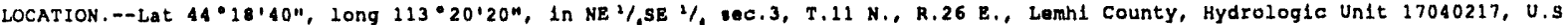
Bureau of Land Management lands on left bank $25 \mathrm{ft}$ downatream from bridge, $0.4 \mathrm{ml}$ upatream from warm creek, $2 \mathrm{mi}$ goutheast of Falrulew guard station, and $16 \mathrm{mi}$ east of Goldberg.

DRAINAGE AREA. $--74.3 \mathrm{ml}$.

PERIOD OF RECORD.- - July 1960 to September 1973.

GAGE, - Water-atage recorder. Altitude of gage is 6,600 ft above sea level, from topographlc map.

REMARKS, - No regulation or diversion above station.

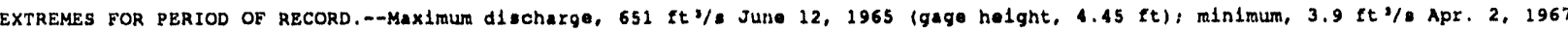
(gage helght, $1.68 \mathrm{tt}$ ).

Sumary of monthly and annual discharges, 1961-73

\begin{tabular}{|c|c|c|c|c|c|c|}
\hline Month & $\begin{array}{l}\text { Max1mum } \\
(\mathrm{ft} J / \mathrm{s})\end{array}$ & $\begin{array}{l}\text { Minlmum } \\
\left(f t^{3} / 8\right)\end{array}$ & $\begin{array}{r}\text { Mean } \\
\left(f t^{2} / s\right)\end{array}$ & $\begin{array}{c}\text { Stan- } \\
\text { dard } \\
\text { devia- } \\
\text { t1:in } \\
\left(\mathrm{ft}^{\prime} / 0\right)\end{array}$ & $\begin{array}{l}\text { Coefil- } \\
\text { clent of } \\
\text { var1- } \\
\text { atlon }\end{array}$ & $\begin{array}{l}\text { Per- } \\
\text { centage } \\
\text { of } \\
\text { annual } \\
\text { runoff }\end{array}$ \\
\hline October & 30 & 14 & 21 & 5.4 & 0.25 & 3.5 \\
\hline November & 31 & 14 & 19 & 4.5 & 0.23 & 3.2 \\
\hline December & 34 & 9.1 & 17 & 6.0 & 0.36 & 2.8 \\
\hline January & 22 & 9.2 & 16 & 3.3 & 0.21 & 2.6 \\
\hline February & 22 & 13 & 17 & 2.6 & 0.16 & 2.8 \\
\hline March & 27 & 13 & 18 & 4.6 & 0.26 & 3.0 \\
\hline Apr 11 & 80 & 17 & 34 & 19 & 0.55 & 5.7 \\
\hline May & 317 & 54 & 149 & 70 & 0.47 & 24.7 \\
\hline June & 400 & 47 & 200 & 103 & 0.52 & 33.1 \\
\hline July & 112 & 22 & 59 & 25 & 0.43 & 9.7 \\
\hline August & 52 & 15 & 29 & 9.5 & 0.32 & 4.9 \\
\hline September & 37 & 16 & 24 & 6.4 & 0.27 & 4.0 \\
\hline Annua 1 & 77 & 24 & 50 & 15 & 0.30 & 100 \\
\hline
\end{tabular}

Magnitude and frequency of instantaneous peak flow, baged on pertod of record 1961-73

\begin{tabular}{|c|c|c|c|c|c|}
\hline $\begin{array}{c}2 \\
50\end{array}$ & $\begin{array}{c}5 \\
204\end{array}$ & $\begin{array}{r}10 \\
10\end{array}$ & $\begin{array}{l}25 \\
41\end{array}$ & $\begin{array}{l}50 \\
21\end{array}$ & $\begin{array}{r}100 \\
1\end{array}$ \\
\hline 377 & 523 & 615 & 728 & 809 & 887 \\
\hline
\end{tabular}

Magnitude and frequency of annual low flow, based on perlod of record 1962-73

\begin{tabular}{|c|c|c|c|c|c|c|}
\hline \multirow{2}{*}{$\begin{array}{l}\text { Perlod } \\
\text { lcon- } \\
\text { ecu- } \\
\text { tive } \\
\text { deys) }\end{array}$} & \multicolumn{6}{|c|}{$\begin{array}{l}\text { Discharge, in } \mathrm{ft}^{\mathrm{s} / \mathrm{s},} \text { for Indlcated } \\
\text { recurrence Interval, in years, and } \\
\text { nonexceedance probability, in percent }\end{array}$} \\
\hline & $\begin{array}{c}2 \\
50\end{array}$ & $\begin{array}{c}5 \\
20\end{array}$ & $\begin{array}{l}10 \\
104\end{array}$ & $\begin{array}{l}20 \\
51\end{array}$ & $\begin{array}{l}501 \\
20\end{array}$ & $\begin{array}{l}100 \\
14\end{array}$ \\
\hline 1 & 13 & 11 & 9.1 & 8.0 & 6.7 & 5.9 \\
\hline 3 & 13 & 11 & 9.4 & 8.3 & 7.0 & 6.2 \\
\hline 7 & 14 & 11 & 9.9 & B. 7 & 7.4 & 6.6 \\
\hline 14 & 14 & 12 & 11 & 9.4 & 8.1 & 7.2 \\
\hline 30 & 15 & 13 & 11 & 9.8 & 8.4 & 7.6 \\
\hline 60 & 16 & 13 & 12 & 10 & 8.9 & 7.9 \\
\hline 90 & 16 & 13 & 12 & 11 & 9.6 & 8.8 \\
\hline 120 & 17 & 14 & 13 & 12 & 11 & 10 \\
\hline 183 & 18 & 15 & 13 & 12 & 11 & 11 \\
\hline
\end{tabular}

Magnitude and frequency of annual high flow, based on pertod of record 1961-73

\begin{tabular}{|c|c|c|c|c|c|c|}
\hline \multirow{2}{*}{$\begin{array}{l}\text { Perlod } \\
\text { (con- } \\
\text { secu- } \\
\text { tive } \\
\text { days) }\end{array}$} & \multicolumn{6}{|c|}{$\begin{array}{l}\text { Discharge, in } \mathrm{ft} 1 / \mathrm{s}, \text { for indlcated } \\
\text { recurrence interval, in years, and } \\
\text { exceedence probability, in percent }\end{array}$} \\
\hline & $50^{2}$ & 20 & $\begin{array}{r}10 \\
10\end{array}$ & $44^{25}$ & 20 & $\begin{array}{l}100 \\
1\end{array}$ \\
\hline 1 & 341 & 479 & 554 & 633 & 683 & 725 \\
\hline 3 & 321 & 452 & 524 & 600 & 647 & 688 \\
\hline 7 & 301 & 427 & 495 & 568 & 612 & 651 \\
\hline 15 & 274 & 390 & 453 & 516 & 555 & 588 \\
\hline 30 & 238 & 338 & 391 & 445 & 477 & 504 \\
\hline 60 & 176 & 252 & 292 & 335 & 360 & 382 \\
\hline 90 & 137 & 191 & 221 & 251 & 269 & 285 \\
\hline
\end{tabular}

Duration table of dally mean flow for perlod of record 1961-73

Lecharge, in fts/s, which was exceeded for indicated percentage of $t$ im

\begin{tabular}{|c|c|c|c|c|c|c|c|c|c|c|c|c|c|c|c|c|}
\hline 14 & 51 & 10 & 251 & 201 & 304 & 404 & 504 & 601 & 70 & 804 & 90 & 951 & 981 & 998 & 99.54 & 99.98 \\
\hline 395 & 225 & 127 & 76 & 54 & 33 & 27 & 23 & 19 & 18 & 16 & 14 & 13 & 12 & 9.0 & 8.5 & 8,0 \\
\hline
\end{tabular}

- Length of record used in calculation may ylold unrellable values for this column. 
ANNUAL MEAN AND MEAN ANNUAL
DISCHAGE, IN CUBIC FEET

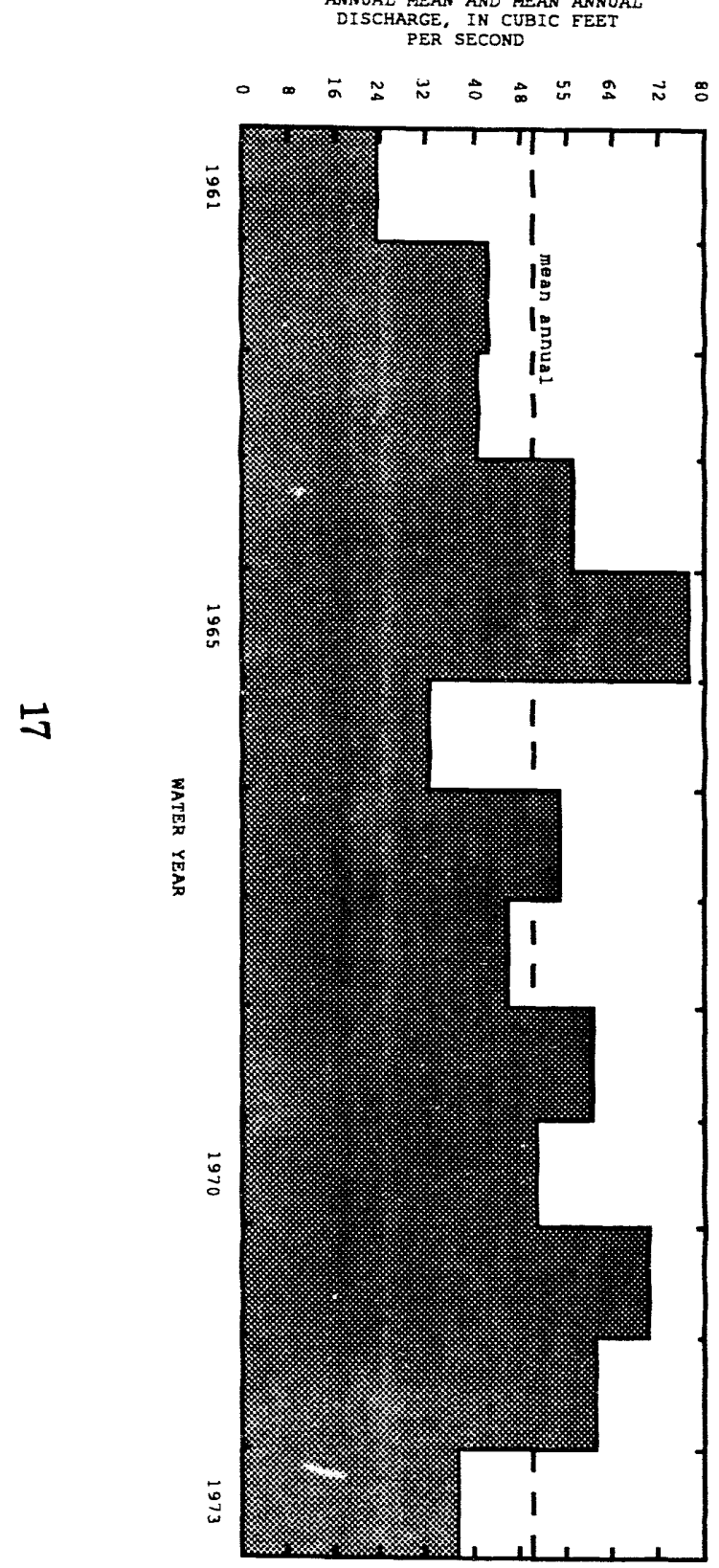

DAILY MEAN DISCHARGE,
IN CUBIC FEET PER SECOND

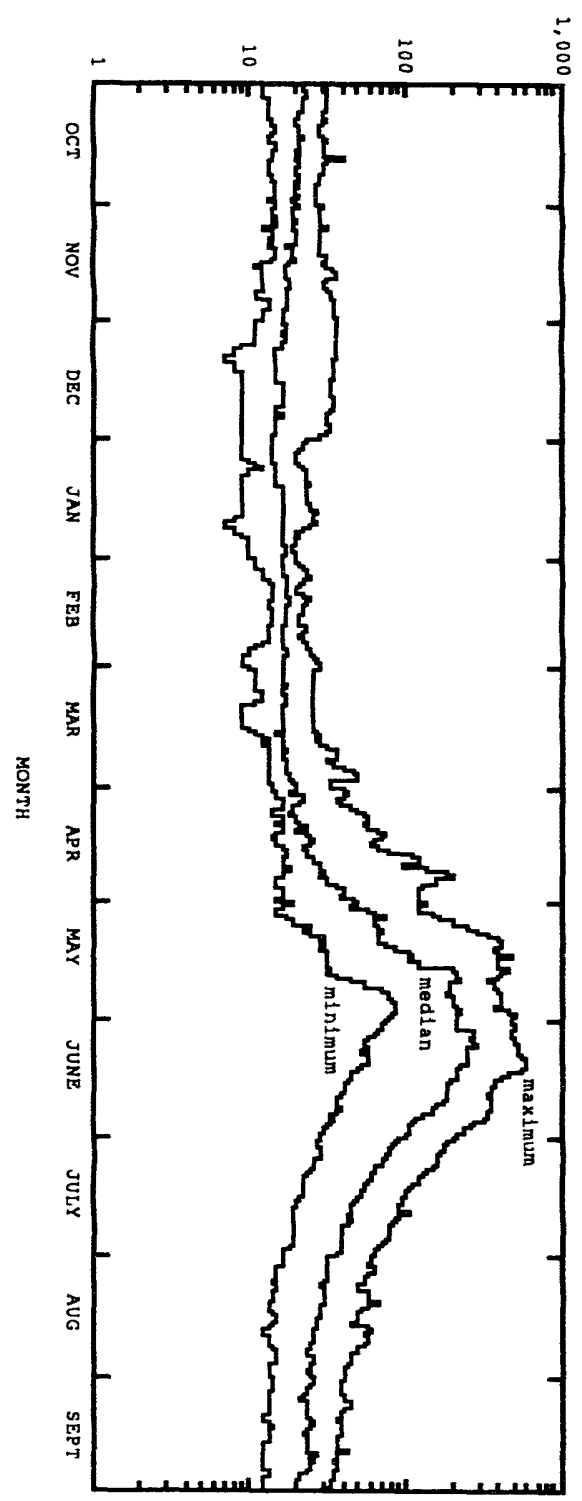

ANNUAL MEAN DISCHARGE,

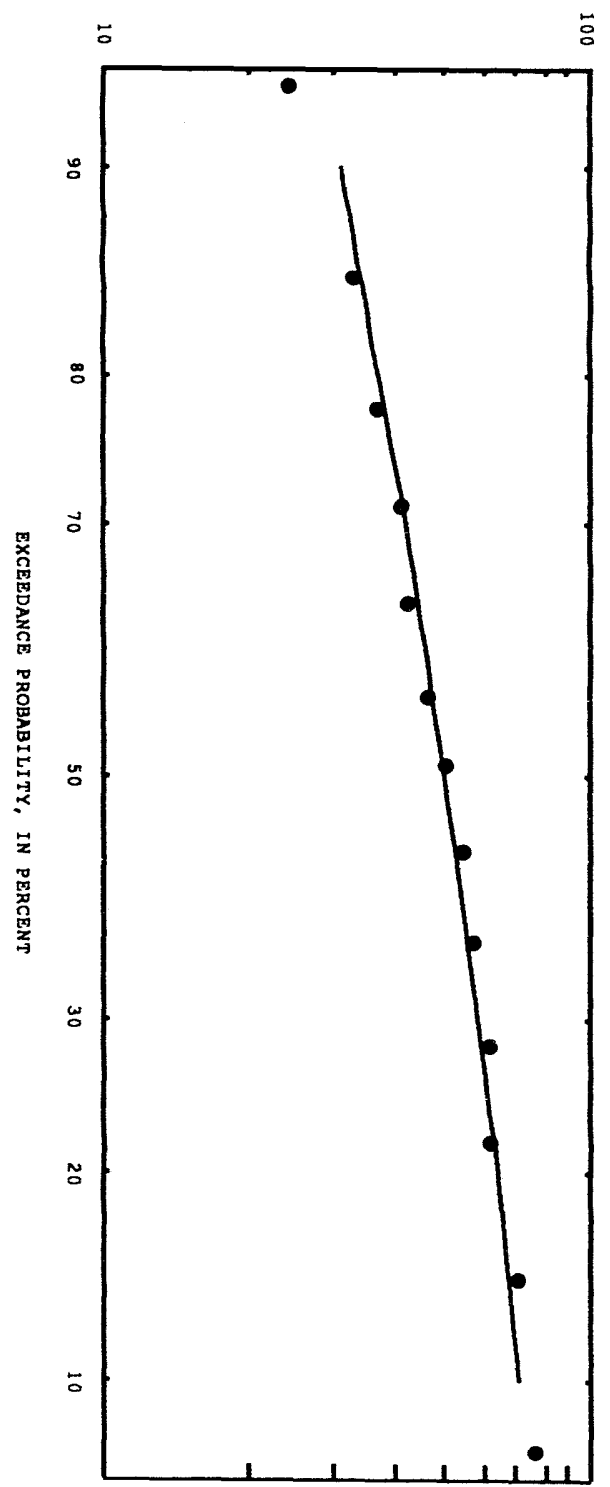


LOCATION,--Lat 44.08119", long $113^{\circ} 14^{\prime} 39^{\prime \prime}$, In NW $1 /$ SE $1 /$ sec.4, T.9 N., R.27 E. Butte County, Hydrolog1c Unit 17050217 , U.S.

Bureas of Land Management lands, on rlght bank at clyde school, $0.6 \mathrm{ml}$ downetream from Met creek, and $27 \mathrm{ml}$ northwest of Howe.

DRAINAGE AREA, $--440 \mathrm{ml}^{*}$, approximately.

PERIOD OF RECORD.--January 1958 to September 1990.

GAGE, - Water-atage recorder. Elevation of gage 1 s $5,800 \mathrm{ft}$ above sea level, from topographlc map.

REMARKs, - D Diversions above gtation for 1rrigation of about 3,800 acres, of which about 2,000 acrea are 1 rrigated by withdrawals from ground water.

EXTREMES FOR PERIOD OF RECORD,--Maximum discharge, $509 \mathrm{ft} \%$, June 16, 1975, gage helght, $3.19 \mathrm{ft}$, but may have been more dur1ng perlod of doubtful gage-helght record in 1958; maximum gage height recorded, 5.99 ft, Feb. 8, 1979 (1ce fam); minlmum discharge recorded, $2.8 \mathrm{ft} / \mathrm{s}$ Dec. 13,1962

summary of monthly and annual discharges, 1959-90

\begin{tabular}{|c|c|c|c|c|c|c|}
\hline Month & $\begin{array}{l}\text { Maximum } \\
\text { (ft } 3 / s)\end{array}$ & $\begin{array}{l}\text { Minimum } \\
\left(\mathrm{ft}^{3} / \mathrm{s}\right)\end{array}$ & $\begin{array}{r}\text { Mean } \\
\left(\mathrm{ft}^{3} / \mathrm{s}\right)\end{array}$ & $\begin{array}{l}\text { stan- } \\
\text { dard } \\
\text { devia- } \\
\text { tlon } \\
\left(\mathrm{ft}^{\prime} / \mathrm{s}\right)\end{array}$ & $\begin{array}{l}\text { coefil- } \\
\text { clent of } \\
\text { vart- } \\
\text { ation }\end{array}$ & $\begin{array}{l}\text { Per- } \\
\text { centage } \\
\text { of } \\
\text { annual } \\
\text { runofe }\end{array}$ \\
\hline October & 101 & 33 & 60 & 17 & 0.29 & 7.2 \\
\hline November & 70 & 17 & 40 & 13 & 0.32 & 4.9 \\
\hline December & 47 & 8.0 & 22 & 7.2 & 0.32 & 2.7 \\
\hline January & 53 & 3.5 & 23 & 9.4 & 0.41 & 2.7 \\
\hline February & 45 & 9.0 & 25 & 8.8 & 0.35 & 3.1 \\
\hline March & 58 & 18 & 37 & 12 & 0.32 & 4.4 \\
\hline Apr 11 & 162 & 24 & 66 & 26 & 0.40 & 7.9 \\
\hline May & 261 & 53 & 139 & 48 & 0.34 & 16.7 \\
\hline June & 354 & 81 & 196 & 76 & 0.39 & 23.5 \\
\hline July & 208 & 34 & 100 & 46 & 0.46 & 12.1 \\
\hline August & 141 & 26 & 63 & 24 & 0.38 & 7.6 \\
\hline September & 128 & 27 & 60 & 21 & 0.35 & 7.2 \\
\hline nnual & 115 & 32 & 69 & 18 & 0.26 & 100 \\
\hline
\end{tabular}

Magnitude and frequency of annual low flow, based on pertod of record 1959-91

\begin{tabular}{|c|c|c|c|c|c|c|}
\hline \multirow{2}{*}{$\begin{array}{l}\text { Perlod } \\
\text { (con- } \\
\text { secu- } \\
\text { tive } \\
\text { days) }\end{array}$} & \multicolumn{6}{|c|}{$\begin{array}{l}\text { Diocharge, in } \mathrm{It}^{2} / \mathrm{s} \text {, for Indlcated } \\
\text { recurrence interval, in years, and } \\
\text { nonexceedance probability, in percent }\end{array}$} \\
\hline & $\begin{array}{c}2 \\
504\end{array}$ & $\begin{array}{c}5 \\
201\end{array}$ & $\begin{array}{l}10 \\
10\end{array}$ & $\begin{array}{l}20 \\
51\end{array}$ & $\begin{array}{l}50 \\
21\end{array}$ & $\begin{array}{l}100 t \\
1\end{array}$ \\
\hline 1 & 16 & 10 & 7.6 & 5.8 & 4.0 & 3.1 \\
\hline 3 & 17 & 11 & B. 0 & 6.0 & 4.1 & 3.2 \\
\hline 7 & 18 & 12 & 8.7 & 6.5 & 4.5 & 3.4 \\
\hline 14 & 20 & 13 & 9.3 & 6.9 & 4.7 & 3,5 \\
\hline 30 & 21 & 13 & 9.8 & 7.2 & 4.9 & 3.6 \\
\hline 60 & 22 & 15 & 11 & 9.0 & 6.7 & 5.4 \\
\hline 90 & 23 & 16 & 13 & 10 & 8.1 & 6.8 \\
\hline 120 & 24 & 18 & 15 & 13 & 11 & 9.2 \\
\hline 183 & 34 & 26 & 23 & 20 & 18 & 16 \\
\hline
\end{tabular}

Magnttude and frequency of annual high flow, based on perlod of record $1959-90$

\begin{tabular}{|c|c|c|c|c|c|c|}
\hline \multirow{3}{*}{$\begin{array}{l}\text { Pertod } \\
\text { (con- } \\
\text { secu- } \\
\text { tive } \\
\text { days) }\end{array}$} & \multicolumn{6}{|c|}{ 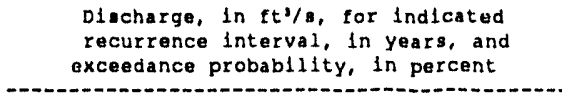 } \\
\hline & 2 & 5 & 10 & 25 & 50 & 1001 \\
\hline & 50 & 208 & 104 & 44 & 24 & 11 \\
\hline 1 & 279 & 371 & 421 & 476 & 511 & 542 \\
\hline 3 & 266 & 357 & 408 & 464 & 501 & 534 \\
\hline 7 & 250 & 335 & 381 & 432 & 465 & 494 \\
\hline 15 & 231 & 310 & 354 & 401 & 432 & 460 \\
\hline 30 & 206 & 277 & 317 & 362 & 392 & 419 \\
\hline 60 & 171 & 229 & 262 & 299 & 324 & 346 \\
\hline 90 & 145 & 192 & 219 & 249 & 268 & 285 \\
\hline
\end{tabular}

Duration table of dally mean flow for perlod of record 1959-90

Dlscharge, in $\mathrm{ft} / \mathrm{s}$, which was exceeded for indicated percentage of time

\begin{tabular}{|c|c|c|c|c|c|c|c|c|c|c|c|c|c|c|c|c|}
\hline 11 & 51 & 104 & 154 & 201 & 301 & 401 & 501 & 601 & 704 & 801 & 904 & 951 & 984 & 991 & 99.51 & 99.98 \\
\hline 322 & 212 & 151 & 117 & 96 & 74 & 60 & 50 & 41 & 32 & 26 & 20 & 16 & 12 & 8.6 & 6.4 & 3.7 \\
\hline
\end{tabular}

- Length of record used in calculation may yleld unrellable values for thls column. 

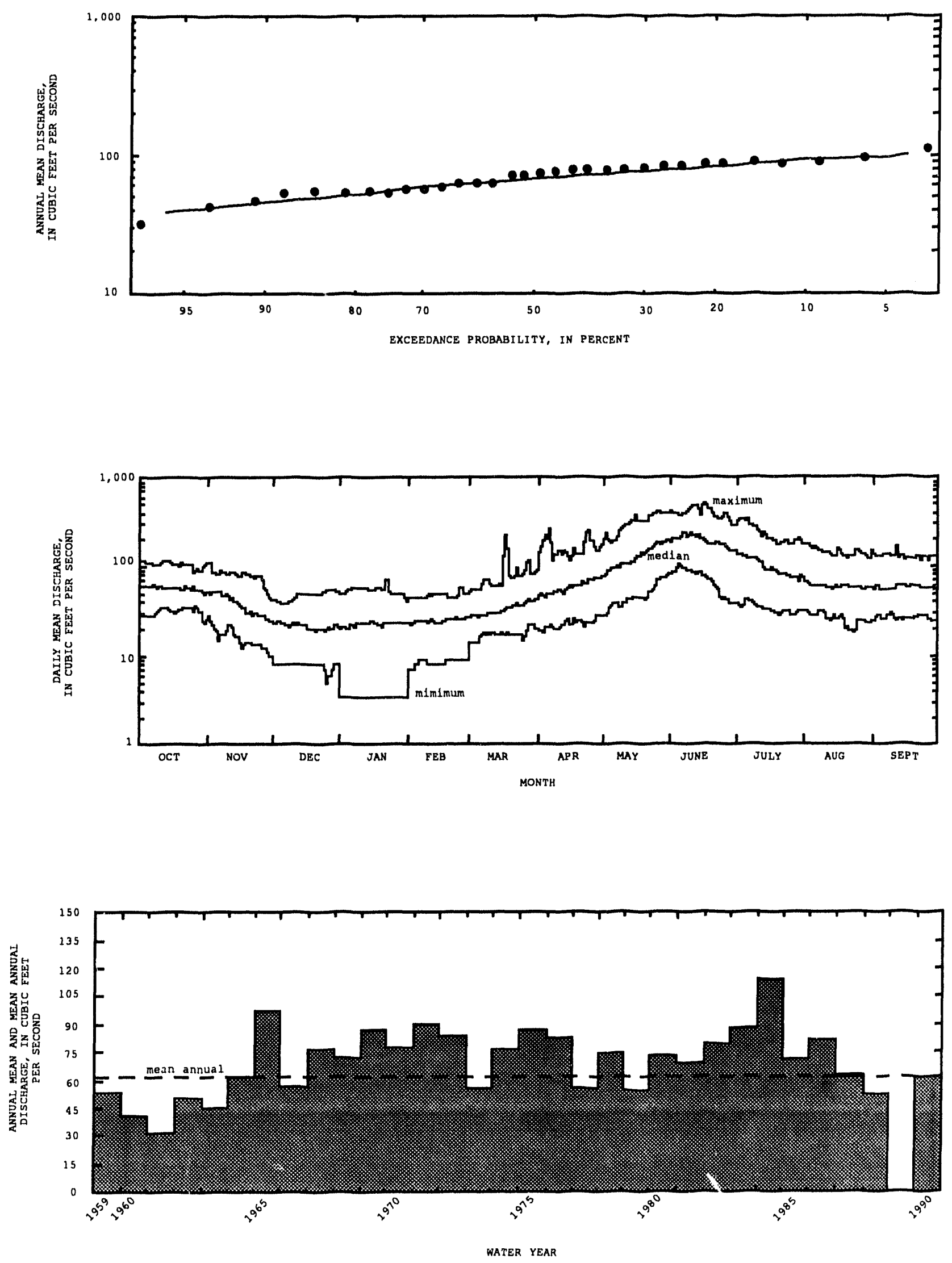


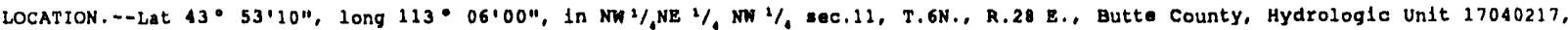
U.s. Bureau of Land Management lands on left bank 0.2 ml upstream from diversion dar of Blaine County Investment Co., and 7 mi northwest of Howe.

DRAINAGE AREA. $--703 \mathrm{mi}$ '.

PERIOD OF RECORD, - April 1921 to September 1981 . May 1985 to September 1990 (no winter recorde prior to October 1940 ). Monthly discharges only for some perlods, published in WSP 1317.

GAGE.--Water-stage recorder. Elevation of gage 1. 5,020 tt above cea level, by barometer. Prior to Sept. 2, 1938 , nonrecording gage at site $120 \mathrm{ft}$ dounstream at datum $1.39 \mathrm{ft}$ hlgher.

REMARKs. - Diversions above station for 1rrigation of about 11,500 acres, of which about 7,600 acres are 1rrigated by withdrawals from ground water (1966 determination). Diversions 1 mi upstrear since Dec. 1984, for winter flood control. Graph of maximum-medianminimum discharge is based on records from 1941-81.

EXTREMES FOR PERIOD OF RECORD.--Maximum discharge, about $450 \mathrm{ft} 3 / \mathrm{s}$ Aug, 11,1936 , during cloudburst, gage helght, $5.4 \mathrm{ft}$, present site and datum from rating curve extended above $220 \mathrm{ft} 3 / \mathrm{s}$; maximum gage helght observed, 6.63 $\mathrm{ft}$, Jan. 23 , 1957 (backwater $\mathrm{from}$ ice): minlmum discharge observed before construction of bypass, $4.1 \mathrm{ft}^{3 / \mathrm{s} \mathrm{Dec}}, 12,1940$.

Summary of monthly and annual discharges, 1941-81, 1986-90

\begin{tabular}{|c|c|c|c|c|c|c|}
\hline Month & $\begin{array}{l}\text { Maximum } \\
(\mathrm{ft} / \mathrm{s})\end{array}$ & $\begin{array}{l}\text { Minfmum } \\
\left(\mathrm{ft}^{3} / \mathrm{s}\right)\end{array}$ & $\begin{array}{r}\text { Mean } \\
\left(\mathrm{ft}^{3} / \mathrm{s}\right)\end{array}$ & $\begin{array}{c}\text { stan- } \\
\text { dard } \\
\text { devis- } \\
\text { tlon } \\
\left(f t^{3} / s\right)\end{array}$ & $\begin{array}{l}\text { Coeffi- } \\
\text { clent of } \\
\text { var1- } \\
\text { ation }\end{array}$ & $\begin{array}{l}\text { Per- } \\
\text { centage } \\
\text { of } \\
\text { annual } \\
\text { runoft }\end{array}$ \\
\hline October & 116 & 46 & 75 & 18 & 0.23 & 8.2 \\
\hline November & 107 & 30 & 58 & 17 & 0.29 & 6.3 \\
\hline December & 57 & 0.00 & 31 & 12 & 0.39 & 3.4 \\
\hline January & 49 & 0.00 & 27 & 13 & 0.48 & 2.9 \\
\hline February & 80 & 0.00 & 35 & 15 & 0.43 & 3.7 \\
\hline March & 112 & 17 & 55 & 22 & 0.40 & 6.0 \\
\hline Apr11 & 174 & 40 & 84 & 25 & 0.30 & 9.2 \\
\hline May & 218 & 73 & 135 & 33 & 0.25 & 14.7 \\
\hline June & 239 & 94 & 164 & 39 & 0.24 & 17.9 \\
\hline July & 195 & 50 & 107 & 33 & 0.31 & 11.6 \\
\hline August & 114 & 44 & 75 & 18 & 0.24 & 8.2 \\
\hline September & 111 & 48 & 72 & 16 & 0.23 & 7.9 \\
\hline Annua l & 106 & 49 & 77 & 15 & 0.20 & 100 \\
\hline
\end{tabular}

Magnitude and frequency of annual low flow based on period of record $1941-81$

\begin{tabular}{|c|c|c|c|c|c|c|}
\hline \multirow{2}{*}{$\begin{array}{l}\text { Period } \\
\text { (con- } \\
\text { gecu- } \\
\text { tive } \\
\text { days) }\end{array}$} & \multicolumn{6}{|c|}{$\begin{array}{l}\text { D1scharge, in } \mathrm{ft} \% \mathrm{~s} \text {, for indlcated } \\
\text { recurrence interval, in years, and } \\
\text { nonexceedance probability, in percent }\end{array}$} \\
\hline & $\begin{array}{c}2 \\
504\end{array}$ & $\begin{array}{c}5 \\
20\end{array}$ & $\begin{array}{l}10 \\
10\end{array}$ & $\begin{array}{l}20 \\
51\end{array}$ & $\begin{array}{l}50 \\
2\end{array}$ & $\begin{array}{l}100 \\
1\end{array}$ \\
\hline 1 & 24 & 16 & 12 & B.7 & 6.0 & 4.6 \\
\hline 3 & 24 & 16 & 12 & 8.8 & 6.1 & 4.6 \\
\hline 7 & 26 & 17 & 12 & 9.1 & 6.2 & 4.6 \\
\hline 14 & 26 & 18 & 14 & 11 & 8.3 & 6.7 \\
\hline 30 & 28 & 20 & 16 & 11 & 10 & 8.5 \\
\hline 60 & 33 & 22 & 16 & 12 & 12 & 10 \\
\hline 90 & 34 & 23 & 17 & 14 & 13 & 11 \\
\hline 120 & 36 & 24 & 17 & 16 & 15 & 14 \\
\hline 183 & 46 & 36 & 31 & 27 & 24 & 21 \\
\hline
\end{tabular}

Magnitude and frequency of annual high flow,

Magnitude and frequency of instantaneous peak flow, based on perlod of record 1941-01, 1986-90

\begin{tabular}{|c|c|c|c|c|c|}
\hline $\begin{array}{c}2 \\
50\end{array}$ & $\begin{array}{c}5 \\
20\end{array}$ & $\begin{array}{r}10 \\
10\end{array}$ & $\begin{array}{l}25 \\
48\end{array}$ & $\begin{array}{l}50 \\
20\end{array}$ & $\begin{array}{r}100 \\
1\end{array}$ \\
\hline 190 & 229 & 262 & 302 & 332 & 361 \\
\hline
\end{tabular}

\begin{tabular}{|c|c|c|c|c|c|c|}
\hline \multirow{2}{*}{$\begin{array}{l}\text { Perlod } \\
\text { (con- } \\
\text { encu- } \\
\text { tive } \\
\text { days) }\end{array}$} & \multicolumn{3}{|c|}{$\begin{array}{l}\text { Discharge, } \\
\text { recurrence } \\
\text { exceedance }\end{array}$} & $\begin{array}{l}\text { In ft'/s, fo } \\
\text { Interval, in } \\
\text { probablitty, }\end{array}$ & \multicolumn{2}{|c|}{$\begin{array}{l}\text { Indlcated } \\
\text { years, and } \\
\text { In percent }\end{array}$} \\
\hline & $\begin{array}{c}2 \\
50 t\end{array}$ & $\begin{array}{c}5 \\
20\end{array}$ & $\begin{array}{l}10 \\
10\end{array}$ & $\begin{array}{l}25 \\
46\end{array}$ & $\begin{array}{l}50 \\
24\end{array}$ & $\begin{array}{c}100 \\
1\end{array}$ \\
\hline 1 & 189 & 226 & 249 & 275 & 294 & 311 \\
\hline 3 & 187 & 222 & 243 & 267 & 283 & 299 \\
\hline 7 & 183 & 218 & 238 & 260 & 276 & 291 \\
\hline 15 & 178 & 212 & 233 & 256 & 272 & 287 \\
\hline 30 & 169 & 204 & 225 & 248 & 265 & 281 \\
\hline 60 & 152 & 185 & 204 & 226 & 241 & 255 \\
\hline 90 & 136 & 165 & 181 & 200 & 212 & 224 \\
\hline
\end{tabular}

Duration table of dally mean flow for perlod of record 1941-81, 1986-90

Discharge, in $\mathrm{ft} \mathrm{s} / \mathrm{s}$, whlch was exceeded for indicated percentage of time

\begin{tabular}{|c|c|c|c|c|c|c|c|c|c|c|c|c|c|c|c|c|}
\hline 11 & 54 & 101 & $15 t$ & 201 & 304 & 404 & 504 & 601 & 708 & 801 & 901 & 951 & 981 & 991 & 99.51 & 99.98 \\
\hline 229 & 180 & 146 & 124 & 110 & 92 & 79 & 68 & 57 & 47 & 37 & 28 & 20 & 0.90 & 0.45 & 0.22 & 0.04 \\
\hline
\end{tabular}

- Length of record used in calculation may yleld unrellable values for this column. 

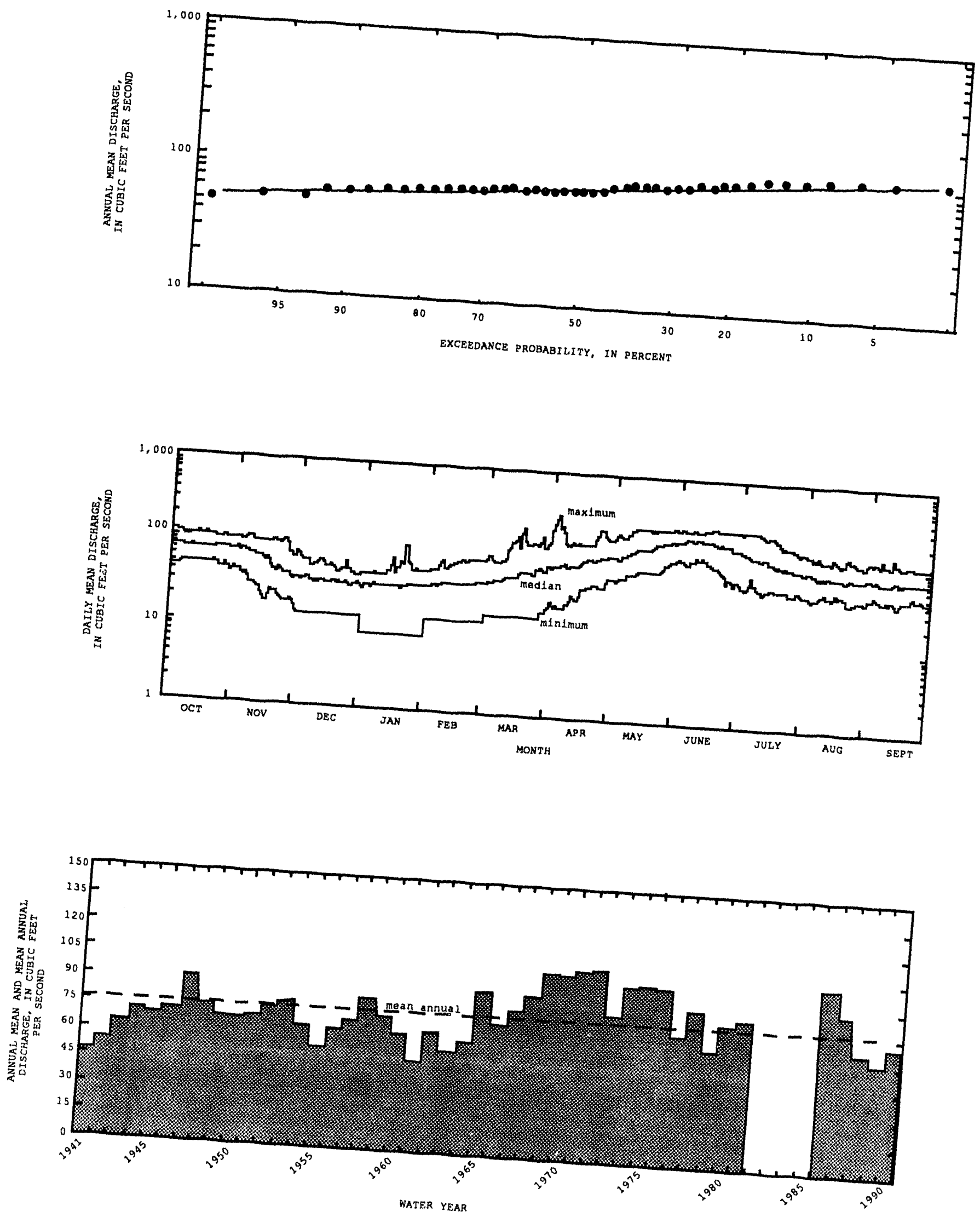
MUD LAKE-LOST RIVER BASIN

23120000 NORTH FORK BIG LOST RIVER AT WILD HORSE, NEAR CHILLY, ID

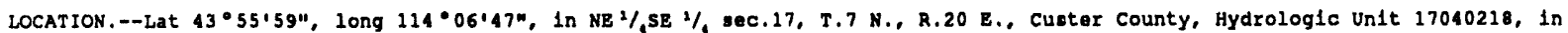
Challs National Forest, on rlght bank $0.2 \mathrm{ml}$ upstream from East Fork, 2 mi downstream from w1ld Horse damsite, and $16 \mathrm{~m} 1$ southwest of chilly.

DRAINAGE AREA. $--114 \mathrm{ml}$.

PERIOD OF 3ECORD.--March 1944 to September 1990. Prior to October 1967, published as "Blg Lost River at w1ld Horse, near Chilly." GAGE. - Water-stage recorder. Elevation of gage 1s 6,820 ft above sea level, from topographle map.

REMARKS.--There are several small ranch diversions upstream for local 1rrigation.

EXTREMES FOR PERIOD OF RECORD.--Maximum discharge, 1,440 ft\%/. May 30, June 1, 1986, gage helght, $5.85 \mathrm{ft}$; minimum, $4.9 \mathrm{ft} 3 / \mathrm{s}$ Feb. 17 , 1988 , gage height, $0.92 \mathrm{ft}$, result of freazeup.

Summary of monthly and annual diacharges, 1945-90

\begin{tabular}{|c|c|c|c|c|c|c|}
\hline Month & $\begin{array}{l}\text { Max1mum } \\
\text { (ft } / \mathrm{s})\end{array}$ & $\begin{array}{l}\text { Minimum } \\
\left(\mathrm{ft}^{3} / \mathrm{s}\right)\end{array}$ & $\begin{array}{r}\text { Mean } \\
\left(f t^{2} / s\right)\end{array}$ & $\begin{array}{c}\text { stan- } \\
\text { dard } \\
\text { devia- } \\
\text { tion } \\
\left(\mathrm{ft}^{3} / \mathrm{s}\right)\end{array}$ & $\begin{array}{l}\text { Coeffi- } \\
\text { clent of } \\
\text { var1- } \\
\text { ation }\end{array}$ & $\begin{array}{l}\text { Per- } \\
\text { centage } \\
\text { of } \\
\text { annual } \\
\text { runoff }\end{array}$ \\
\hline October & 64 & 22 & 40 & 11 & 0.27 & 3.2 \\
\hline November & 117 & 19 & 33 & 14 & 0.44 & 2.6 \\
\hline December & $B 8$ & 17 & 27 & 11 & 0.40 & 2.1 \\
\hline January & $B O$ & 15 & 25 & 9.8 & 0.40 & 2.0 \\
\hline Eebruary & 71 & 15 & 22 & 8.1 & 0.37 & 1.8 \\
\hline March & 62 & 14 & 23 & 8.4 & 0.37 & 1.8 \\
\hline Apr 11 & 153 & 17 & 65 & 35 & 0.54 & 5.2 \\
\hline May & 584 & 66 & 284 & 121 & 0.43 & 22.7 \\
\hline June & 848 & 156 & 415 & 162 & 0.39 & 33.2 \\
\hline July & 506 & 57 & 196 & 101 & 0.51 & 15.7 \\
\hline August & 178 & 30 & 72 & 29 & 0.41 & 5.8 \\
\hline September & 122 & 22 & 48 & 20 & 0.42 & 3.9 \\
\hline Annual & 184 & 52 & 104 & 32 & 0.31 & 100 \\
\hline
\end{tabular}

Magnitude and frequency of Instantaneous peak flow, based on perlod of record $1945-90$

\begin{tabular}{|c|c|c|c|c|c|}
\hline $\begin{array}{c}2 \\
504\end{array}$ & $\begin{array}{c}5 \\
20 t\end{array}$ & $\begin{array}{r}10 \\
104\end{array}$ & $\begin{array}{l}25 \\
44\end{array}$ & $\begin{array}{l}50 \\
24\end{array}$ & $\begin{array}{r}100 \\
1\end{array}$ \\
\hline 732 & 1,020 & 1,210 & 1,440 & 1,610 & 1,780 \\
\hline
\end{tabular}

Magnitude and frequency of annual low flow, based on perlod of record 1945-91

\begin{tabular}{|c|c|c|c|c|c|c|}
\hline \multirow{2}{*}{$\begin{array}{l}\text { Perlod } \\
\text { (con- } \\
\text { secu- } \\
\text { tive } \\
\text { days) }\end{array}$} & \multicolumn{6}{|c|}{$\begin{array}{l}\text { Discharge, in ft'/s, for findicated } \\
\text { recurrence interval, in years, and } \\
\text { nonexceedance probability, in percent }\end{array}$} \\
\hline & $\begin{array}{c}2 \\
504\end{array}$ & $\begin{array}{c}5 \\
20\end{array}$ & $\begin{array}{l}10 \\
10\end{array}$ & $\begin{array}{l}20 \\
54\end{array}$ & $\begin{array}{l}50 \\
24\end{array}$ & $\begin{array}{l}100 \\
1\end{array}$ \\
\hline 1 & 16 & 14 & 13 & 12 & 11 & 9.9 \\
\hline 3 & 17 & 15 & 13 & 12 & 11 & 11 \\
\hline 7 & 17 & 15 & 14 & 13 & 12 & 12 \\
\hline 14 & 18 & 16 & 15 & 14 & 13 & 13 \\
\hline 30 & 19 & 16 & 15 & 15 & 14 & 14 \\
\hline 60 & 19 & 18 & 17 & 17 & 17 & 17 \\
\hline 90 & 20 & 18 & 18 & 18 & $: 8$ & 18 \\
\hline 120 & 21 & 19 & 18 & 18 & 18 & 18 \\
\hline 183 & 25 & 22 & 21 & 20 & 20 & 20 \\
\hline
\end{tabular}

Magnitude and trequency of annual high flow. based on period of record $1945-9$

\begin{tabular}{|c|c|c|c|c|c|c|}
\hline \multirow{2}{*}{$\begin{array}{l}\text { Period } \\
\text { (con- } \\
\text { secu- } \\
\text { t1ve } \\
\text { days) }\end{array}$} & \multicolumn{4}{|c|}{ 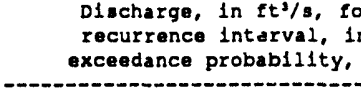 } & \multicolumn{2}{|c|}{$\begin{array}{l}\text { Indicated } \\
\text { years, and } \\
\text { n percent }\end{array}$} \\
\hline & $\begin{array}{c}2 \\
50\end{array}$ & $\begin{array}{c}5 \\
208\end{array}$ & $\begin{array}{l}10 \\
10\end{array}$ & $\begin{array}{l}25 \\
4 t\end{array}$ & $\begin{array}{l}50 \\
24\end{array}$ & $\begin{array}{c}100 \\
1\end{array}$ \\
\hline 1 & 670 & 937 & 1,120 & 1,340 & 1,510 & 1,690 \\
\hline 3 & 628 & 881 & 1,050 & 1,270 & 1,440 & 1,610 \\
\hline 7 & 574 & 809 & 968 & 1,170 & 1,320 & 1,480 \\
\hline 15 & 511 & 719 & 857 & 1,030 & 1,160 & 1,290 \\
\hline 30 & 411 & 608 & 715 & 847 & 942 & 1,040 \\
\hline 60 & 358 & 490 & 573 & 673 & 744 & 812 \\
\hline 90 & 291 & 396 & 459 & 534 & 586 & 636 \\
\hline
\end{tabular}

Duration table of dally mean flow for perlod of record 1945-90

Discharge, in $\mathrm{ft} / \mathrm{s}$, which was exceeded for indicated percentage of time

\begin{tabular}{|c|c|c|c|c|c|c|c|c|c|c|c|c|c|c|c|c|}
\hline 14 & 54 & 104 & 154 & 204 & 304 & 408 & 508 & 604 & 708 & 804 & 90 & 951 & 981 & 994 & 99.51 & 99.91 \\
\hline & 435 & 305 & 212 & 148 & 75 & 50 & 38 & 31 & 26 & 23 & 20 & 18 & 17 & 15 & 14 & 13 \\
\hline
\end{tabular}

- Length of record used In calculation may yield unrellable values for this column. 

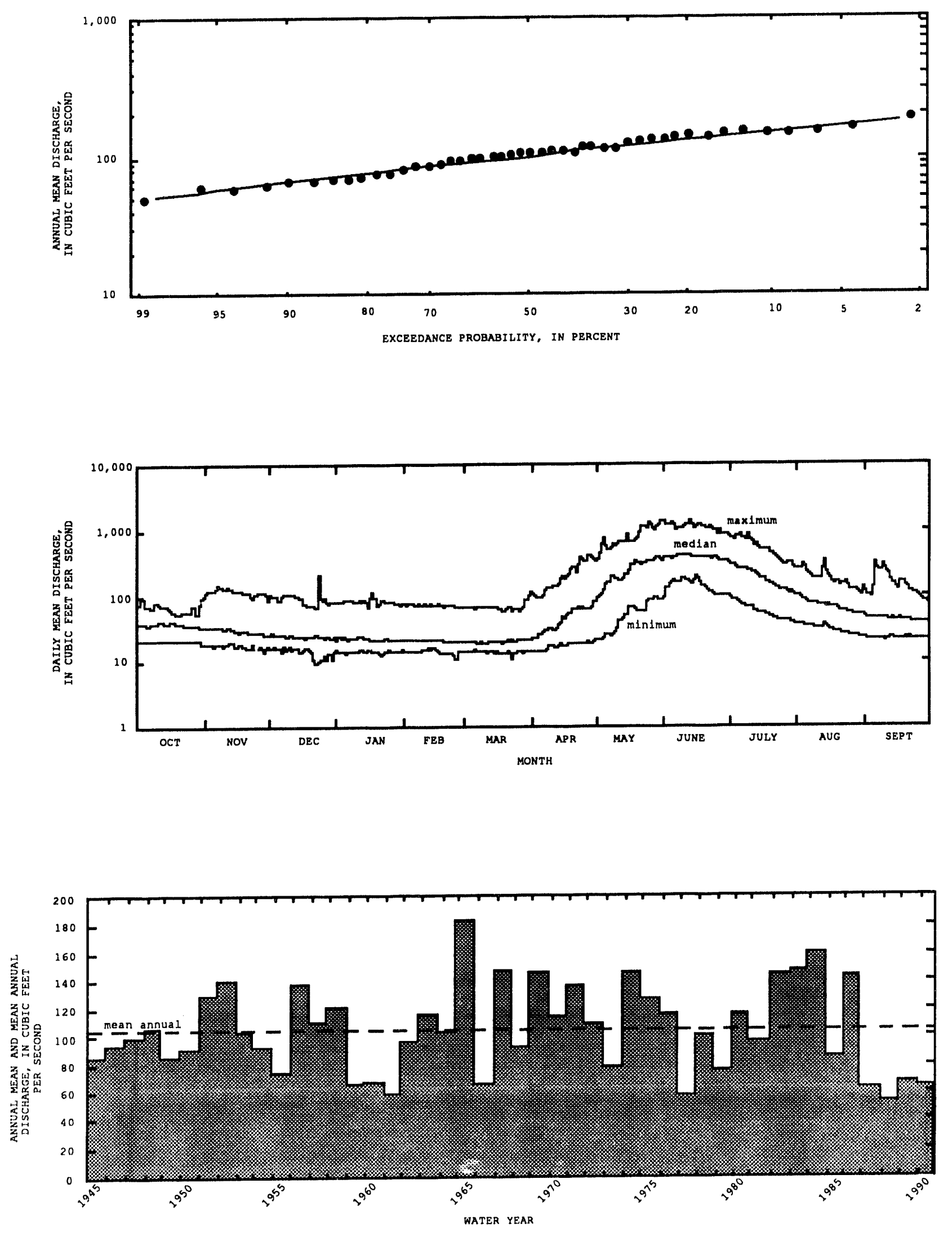
13120500 BIG LOST RIVER AT HOWELL RANCH, MEAR CHILLY, ID

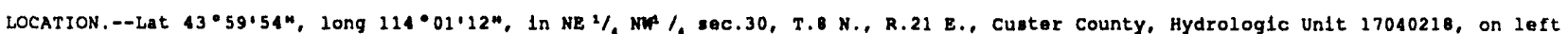
bank at Howell Ranch, $2.1 \mathrm{ml}$ downstream from Burnt Creek, $7.7 \mathrm{ml}$ downstream from East Fork, 9 ml southwest of Chilly, and 21 mi northwest of Mackay.

DRAINAGE AREA. $--450 \mathrm{~m} \mathbf{1}^{\prime}$.

PERIOD OF RECORD.--April 1904 to November 1914, May 1920 to September 1990 (no winter records 1904, 1906-14, 1920-48).

GAGE. --Water-stage recorder. Datum of gage 1. 6,621.95 ft above sea level, from topographic map. See wSP 1737 for h1story of changes prior to June 11,1920 .

REMARKS.-No regulation. Diversions above atation for irrigation of about 3,000 acres (1966 determination).

EXTREMES FOR RERIOD OF RECORD.--Maxtmum dlecharge, 4,420 ft'/s, May 25, 1967, gage helght, $6.02 \mathrm{ft}$; minlmum observed, $19 \mathrm{ft} / \mathrm{s}$ Dec. 12, 1939 (d1 scharge measurement).

Summary of monthly and annual discharges, 1905, 1949-90

\begin{tabular}{|c|c|c|c|c|c|c|}
\hline Month & $\begin{array}{l}\text { Max1mum } \\
(\mathrm{ft} / / \mathrm{s})\end{array}$ & $\begin{array}{l}\text { Minfmum } \\
(\mathrm{ft} / \mathrm{s})\end{array}$ & $\begin{array}{l}\text { Mean } \\
\left(f t^{2} / s\right)\end{array}$ & $\begin{array}{c}\text { stan- } \\
\text { dard } \\
\text { devia- } \\
\text { tion } \\
\left(\mathrm{ft}^{3} / \mathrm{s}\right)\end{array}$ & $\begin{array}{l}\text { Coeffi- } \\
\text { clent of } \\
\text { var1- } \\
\text { ation }\end{array}$ & $\begin{array}{l}\text { Per- } \\
\text { centage } \\
\text { of } \\
\text { annual } \\
\text { runoft }\end{array}$ \\
\hline Oct ober & 223 & 66 & 129 & 38 & 0.30 & 3.3 \\
\hline Nol ember & 373 & 61 & 111 & 48 & 0.43 & 2.9 \\
\hline December & 278 & 57 & 92 & 35 & 0.38 & 2.4 \\
\hline January & 245 & 51 & 86 & 31 & 0.36 & 2.3 \\
\hline February & 218 & 49 & 80 & 26 & 0.33 & 2.1 \\
\hline March & 194 & 47 & 82 & 25 & 0.30 & 2.1 \\
\hline April & 448 & 64 & 191 & 92 & 0.48 & 4.9 \\
\hline May & 1,880 & 200 & 814 & 373 & 0.46 & 21.0 \\
\hline June & 2,350 & 487 & 1.290 & 515 & 0.40 & 33.2 \\
\hline Ju2y & 1,470 & 153 & 616 & 351 & 0.57 & 15.9 \\
\hline August & 586 & 84 & 230 & 110 & 0.48 & 5.9 \\
\hline September & 378 & 67 & 156 & 67 & 0.43 & 4.0 \\
\hline Annua 1 & 538 & 168 & 323 & 105 & 0.33 & 100 \\
\hline
\end{tabular}

Magnitude and frequency of instantaneous peak flow, based on pertod of record 1905, 1949-90

\begin{tabular}{|c|c|c|c|c|c|}
\hline $\begin{array}{c}2 \\
50\end{array}$ & $\begin{array}{c}5 \\
20\end{array}$ & $\begin{array}{r}10 \\
10\end{array}$ & $\begin{array}{l}25 \\
41\end{array}$ & $\begin{array}{l}50 \\
21\end{array}$ & $\begin{array}{r}100 \\
1\end{array}$ \\
\hline 2,150 & 2,960 & 3.450 & 3,990 & 4,360 & 4,69 \\
\hline
\end{tabular}

Magnitude and frequency of annual low flow, based on period of record 1949-90

\begin{tabular}{|c|c|c|c|c|c|c|}
\hline \multirow{2}{*}{$\begin{array}{l}\text { Perlod } \\
\text { (con- } \\
\text { secu- } \\
\text { tive } \\
\text { days) }\end{array}$} & \multicolumn{6}{|c|}{$\begin{array}{l}\text { D1scharge, in ft } 1 / \mathrm{s} \text {, for lndicated } \\
\text { recurrence interval, in years, and } \\
\text { nonexceedance probability, in percent }\end{array}$} \\
\hline & $\begin{array}{c}2 \\
504\end{array}$ & $\begin{array}{c}5 \\
20\end{array}$ & $\begin{array}{l}10 \\
104\end{array}$ & $\begin{array}{l}20 \\
51\end{array}$ & $\begin{array}{l}50 \\
21\end{array}$ & $\begin{array}{l}100 \\
14\end{array}$ \\
\hline 1 & 58 & 48 & 44 & 40 & 36 & 34 \\
\hline 3 & 60 & 52 & 48 & 45 & 42 & 40 \\
\hline 7 & 63 & 54 & 50 & 47 & 44 & 42 \\
\hline 14 & 65 & 55 & 51 & 48 & 45 & 44 \\
\hline 30 & 69 & 58 & 54 & 51 & 47 & 45 \\
\hline 60 & 70 & 61 & 58 & 56 & 55 & 55 \\
\hline 90 & 73 & 64 & 61 & 59 & 58 & 58 \\
\hline 120 & 76 & 66 & 64 & 62 & 61 & 61 \\
\hline 183 & 89 & 75 & 70 & 68 & 65 & 64 \\
\hline
\end{tabular}

Magnitude and frequency of annual high flow. based on period of record 1905, 1949-90

\begin{tabular}{|c|c|c|c|c|c|c|}
\hline \multirow{3}{*}{$\begin{array}{l}\text { Perlod } \\
\text { (con- } \\
\text { secu- } \\
\text { tive } \\
\text { days) }\end{array}$} & \multicolumn{6}{|c|}{$\begin{array}{l}\text { Discharge, in } t t^{\prime} / s, \text { for indlcated } \\
\text { recurrence Interval, in years, and } \\
\text { exceedance probablilty, in percent }\end{array}$} \\
\hline & 2 & 5 & 10 & 25 & 50 & 100 \\
\hline & 504 & 201 & 10 & $4 t$ & 21 & 14 \\
\hline 1 & 2,130 & 2,860 & 3,330 & 3,900 & 4,310 & 4,650 \\
\hline 3 & 1,980 & 2,680 & 3,130 & 3,690 & 4,110 & 4,520 \\
\hline 7 & 1,780 & 2,450 & 2,890 & 3,440 & 3,840 & 4,250 \\
\hline 15 & 1,580 & 2,200 & 2,600 & 3.100 & 3,470 & 3,840 \\
\hline 30 & 1,360 & 1,880 & 2,210 & 2,630 & 2,930 & 3,230 \\
\hline 60 & 1,070 & 1,500 & 1,790 & 2,130 & 2,390 & 2,640 \\
\hline 90 & 867 & 1,210 & 1,440 & 1,710 & 1,900 & 2,100 \\
\hline
\end{tabular}

Duration table of dally mean flow for period of record 1905, 1949-90

\begin{tabular}{|c|c|c|c|c|c|c|c|c|c|c|c|c|c|c|c|c|}
\hline 11 & 54 & 101 & 151 & 20 & 304 & 401 & 504 & 601 & 704 & 804 & 901 & 95 & 984 & 991 & $99.5 t$ & 99.91 \\
\hline 2,350 & 1,370 & 911 & 617 & 435 & 228 & 158 & 125 & 105 & 89 & 79 & 68 & 60 & 54 & 50 & 48 & 43 \\
\hline
\end{tabular}

- Length of record used in calculation may yleld unzellable values for this colums. 

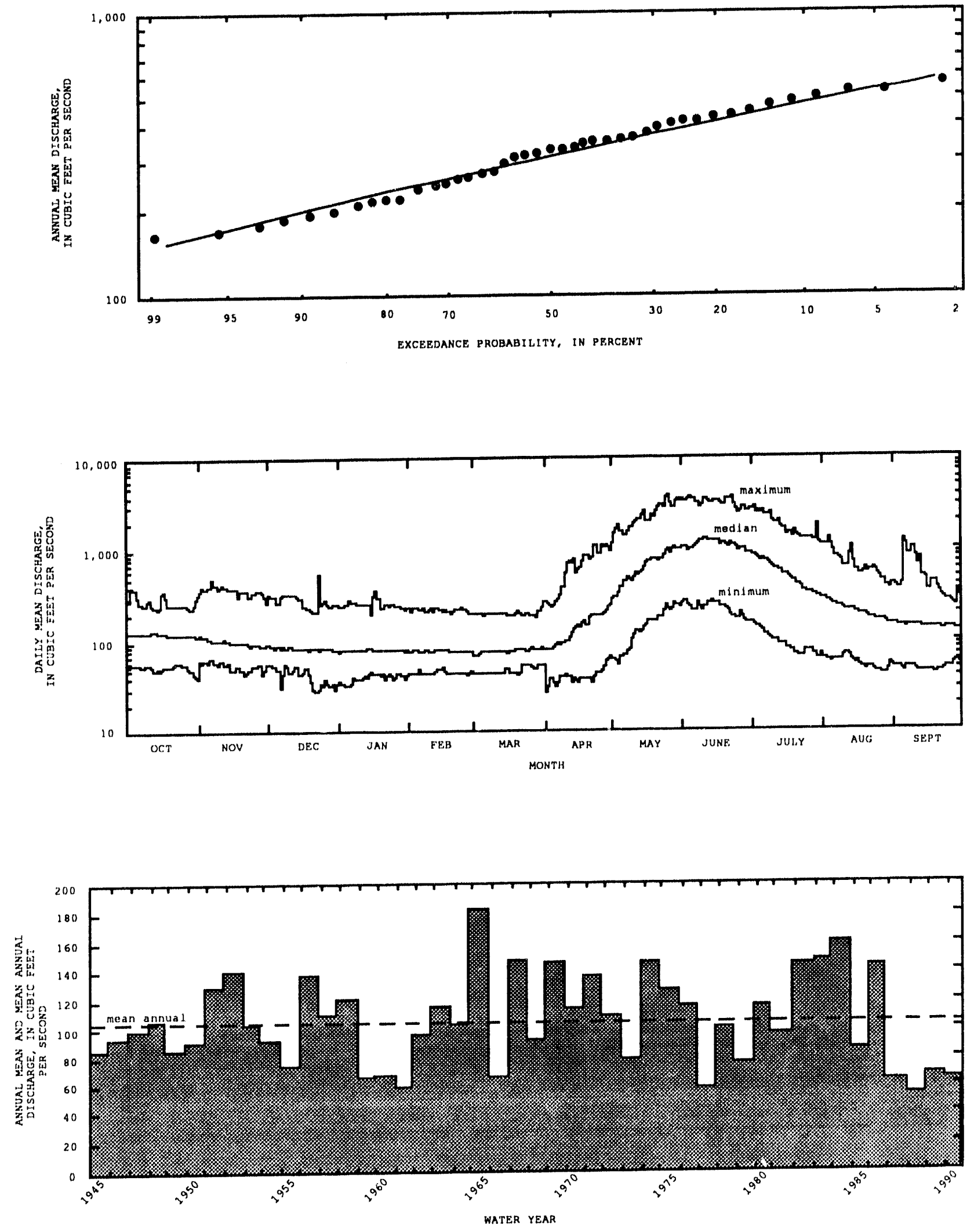
13127000 BIG LOST RIVER BELOW MACKAY RESERVOIR, NEAR MACKAY, ID

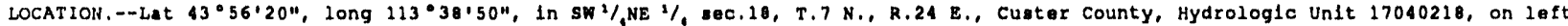
bank $1.4 \mathrm{ml}$ downatream from head of sharp Ditch, $1.6 \mathrm{ml}$ downstream from Mackay Reservolr, and $2.5 \mathrm{ml}$ northwest of Mackay.

DRAINAGE AREA. $--813 \mathrm{~m} \mathrm{~L}^{2}$.

PERIOD OF RECORD.--December 1903 to Auguat 1906, and May 1912 to March 1915 (publiohed as "near Mackay"), January 1919 to September 1990.

GAGE.--Water-gtage recorder. Datum of gage $1 \mathrm{~s} 5,946.39 \mathrm{ft}$ above sea level, from topographle map. Nonrecording gage prlor to May 12, 1912, and June 5, 1912, to Apr. 28, 1913, at altes with1n 1 m1 upstream at different datums, May 12 to June 4 , 1912 , at site $1.5 \mathrm{ml}$ upstream (above Sharp D1tch) at different datum, Apr. 29, 1913, to Mar. 15, 1915, at B1te $1 \mathrm{ml}$ downstream (below Streeter Ditch) at different datum.

REMARKS. -Flow completely regulated by Mackay Reservolr. Sharp Ditch 1 s only diversion between station and reservolr; about 12,700 acres of land are irrigated by diversions from river and tributartes above reservolr by surface diversions, and 10,200 acres irrlgated by sublrigation.

EXTREMES FOR PERIOD OF RECORD.--Maximum d1scharge, 2,990 ft'/s June 10, 1921, June 6, 1986, maximum gage helght, 6.08 ft, June 6, $1986 ; \mathrm{minlmum}, 16 \mathrm{ft} / \mathrm{s}$ Oct. 27, 1967, gage helght, $1.11 \mathrm{ft}$.

summary of monthly and annual d1 acharges, 1905, 1913-14, 1920-90

\begin{tabular}{|c|c|c|c|c|c|c|}
\hline Month & $\begin{array}{l}\text { Maximum } \\
\text { (ft } 1 / s)\end{array}$ & $\begin{array}{l}\text { Minimum } \\
\left(\mathrm{ft}^{3} / \mathrm{s}\right)\end{array}$ & $\begin{array}{l}\text { Mean } \\
\left(\mathrm{ft}^{3} / \mathrm{s}\right)\end{array}$ & $\begin{array}{l}\text { stan- } \\
\text { dard } \\
\text { devla- } \\
\text { tlon } \\
\left(\mathrm{ft}^{\prime} / \mathrm{s}\right)\end{array}$ & $\begin{array}{l}\text { Coetel- } \\
\text { clent of } \\
\text { var1- } \\
\text { ation }\end{array}$ & $\begin{array}{l}\text { Per- } \\
\text { centage } \\
\text { of } \\
\text { annual } \\
\text { runoff }\end{array}$ \\
\hline October & 487 & 60 & 166 & 75 & 0.45 & 4.5 \\
\hline November & 660 & 45 & 104 & 83 & 0.79 & 2.8 \\
\hline December & 476 & 59 & 112 & 63 & 0.56 & 3.0 \\
\hline January & 292 & 75 & 123 & 42 & 0.34 & 3.3 \\
\hline February & 304 & 82 & 130 & 39 & 0.30 & 3.5 \\
\hline March & 544 & 94 & 148 & 70 & 0.47 & 4.0 \\
\hline Apr 11 & 516 & 93 & 164 & 87 & 0.53 & 4.4 \\
\hline May & 1,190 & 116 & 494 & 252 & 0.51 & 13,3 \\
\hline June & 2,010 & 203 & 955 & 405 & 0.42 & 25.7 \\
\hline July & 1,470 & 127 & 678 & 274 & 0.40 & 18.2 \\
\hline August & 895 & 113 & 417 & 183 & 0.44 & 11.2 \\
\hline Sept ember & 635 & 100 & 225 & 96 & 0.43 & 6.1 \\
\hline Annual & 658 & 128 & 311 & 102 & 0.33 & 100 \\
\hline
\end{tabular}

Magnitude and frequency of Instantaneoug peak flow, based on perlod of record 1905, 1913-14, 1920-90

Discharge, in $\mathrm{ft} / \mathrm{s}$, for Indicated recurrence interval. in years, and exceedance probablitty, in percent

$\begin{array}{cccccc}2 & 5 & 10 & 25 & 50 & 100 \\ 504 & 204 & 104 & 41 & 28 & 11 \\ 1,440 & 2,030 & 2,400 & 2,840 & 3,160 & 3,460\end{array}$

Magnitude and frequency of annual low tlow, based on perlod of record 1905-06, 1914, 1920-91

\begin{tabular}{|c|c|c|c|c|c|c|}
\hline \multirow{2}{*}{$\begin{array}{l}\text { Perlod } \\
\text { (con- } \\
\text { secu- } \\
\text { tive } \\
\text { days) }\end{array}$} & \multicolumn{6}{|c|}{ 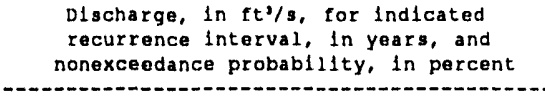 } \\
\hline & $\begin{array}{c}2 \\
50\end{array}$ & $\begin{array}{c}5 \\
208\end{array}$ & $\begin{array}{l}10 \\
104\end{array}$ & $\begin{array}{l}20 \\
51\end{array}$ & $\begin{array}{l}50 \\
24\end{array}$ & $\begin{array}{l}100 \\
11\end{array}$ \\
\hline 1 & 55 & 38 & 31 & 26 & 22 & 20 \\
\hline 3 & 58 & 40 & 33 & 28 & 24 & 21 \\
\hline 7 & 61 & 43 & 36 & 31 & 26 & 23 \\
\hline 14 & 64 & 47 & 40 & 35 & 31 & 28 \\
\hline 30 & 69 & 53 & 48 & 45 & 42 & 40 \\
\hline 60 & 77 & 62 & 58 & 55 & 52 & 51 \\
\hline 90 & 87 & 71 & 65 & 62 & 59 & 58 \\
\hline 120 & 93 & 77 & 72 & 70 & 67 & 67 \\
\hline 183 & 115 & 97 & 92 & 88 & 86 & 85 \\
\hline
\end{tabular}

Magnitude and frequency of annual high flow, based on perlod of record 1905, 1913-14, 1920-90

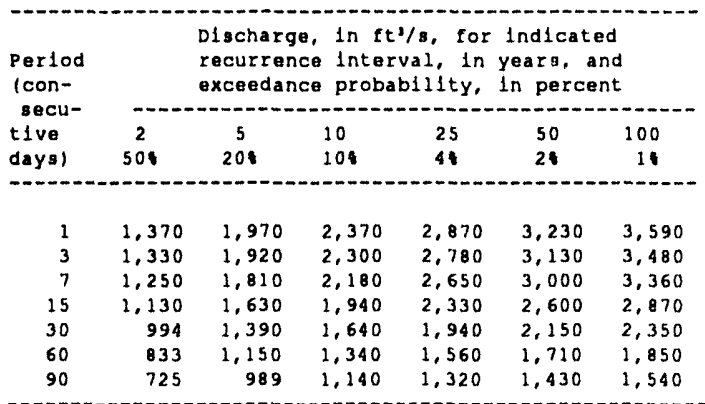

Duration table of dally mean flow for period of record 1905, 1913-14, 1920-90

Diacharge, In $\mathrm{ft}^{\mathrm{s}} / \mathrm{s}$, which was exceeded for indlcated percentage of time

\begin{tabular}{|c|c|c|c|c|c|c|c|c|c|c|c|c|c|c|c|c|}
\hline 11 & $5 t$ & 108 & 151 & 201 & 301 & 401 & 501 & 601 & 703 & 801 & 908 & 951 & 981 & 991 & 99.51 & 99.98 \\
\hline 78 & 990 & 763 & 628 & 513 & 294 & 195 & 160 & 130 & 115 & 101 & 82 & 67 & 55 & 48 & 42 & 29 \\
\hline
\end{tabular}

- Length of record used in calculation may yleld unreliable values for this column. 

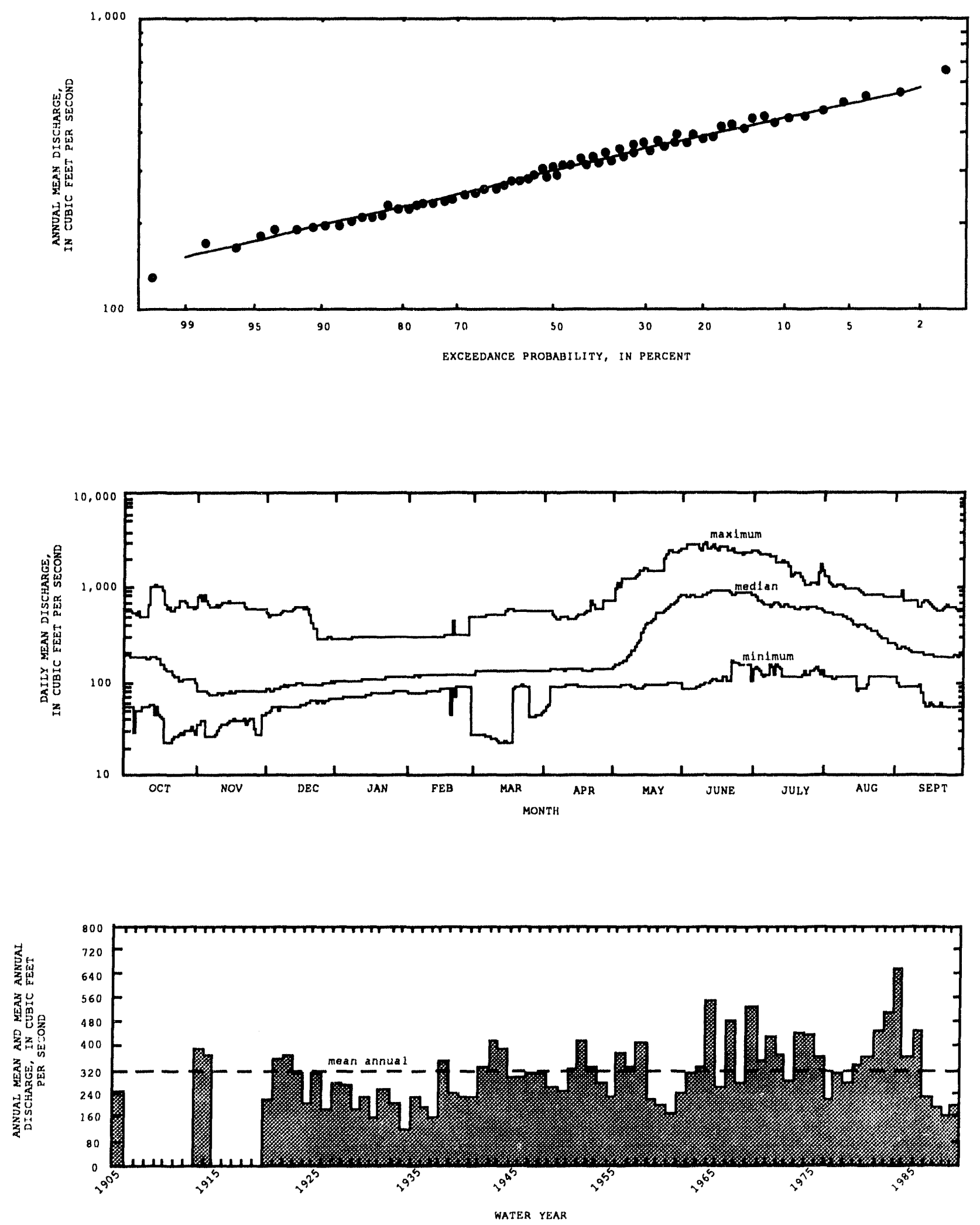
LOCATION,--Lat $43^{\circ} 57.57 \%$, long $113 \cdot 34140 "$, In NW $1 /$ sW $1 /$ sec.2, T.7 N., R.24 E., Custer County, Hydrolog1c Unit 17040218, Cha1118 National Forest, on rlght bank at abandoned powerplant ite, approximately 1,000 ft upstream from the heading of Nielson diversion, and $3.9 \mathrm{ml}$ northeast of Mackay.

DRAINAGE AREA, $--8.26 \mathrm{ml}$ \%

PERIOD OF RECORD. - -Water years 1963, 1964-66, August 1966 to September 1973, October 1979 to September 1990 . Comb1nat1on of d1scharge records for Clark Ditch near Mackay and Cedar Creek (below powerplant) near Mackay for May 1920 to September 1922 (seagonal records only) is equivalent to thls record.

GAGE.--Water-stage recorder. Alt1tude of gage 1g 6,800 ft above sea level, from topographlc map. May 1, 1920, to Oct. 21, 1922, nonrecording gage at present site at different datums. Sept. 26, 1963, to Aug, 13, 1966, crest-stage gage at slte 20 ft downstream at datum $9.32 \mathrm{ft}$ lower.

REMARKS. - No regulation or diversion above station.

EXTREMES FOR PERIOD OF RECORD.--Maxlmum discharge, $310 \mathrm{ft} / \mathrm{s}$ June 22,1982 , gage helght, $3.10 \mathrm{ft}$. Minimum dibcharge observed, 0.4 $\mathrm{ft}^{\prime} / \mathrm{s}$ Nov. 21 to Dec. 1, 1921 , gage height, $0.18 \mathrm{ft}$, site and datum then in use.

Summary of monthly and annual discharqes, 1967-73, 1980-84

\begin{tabular}{|c|c|c|c|c|c|c|}
\hline Month & $\begin{array}{l}\text { Maximum } \\
(\mathrm{ft} / \mathrm{s})\end{array}$ & $\begin{array}{l}\text { Minlmum } \\
\left(f t^{\prime} / s\right)\end{array}$ & $\begin{array}{c}\text { Mean } \\
\left(f t^{2} / s\right)\end{array}$ & $\begin{array}{c}\text { stan- } \\
\text { dard } \\
\text { devia } \\
\text { tlon } \\
\left(\mathrm{ft}^{3} / \mathrm{s}\right)\end{array}$ & $\begin{array}{l}\text { Coeft } 1- \\
\text { clent of } \\
\text { var1- } \\
\text { atlon }\end{array}$ & $\begin{array}{l}\text { Per- } \\
\text { centage } \\
\text { of } \\
\text { annual } \\
\text { runoff }\end{array}$ \\
\hline October & 27 & 6.8 & 12 & 5.4 & 0.44 & 4.7 \\
\hline November & 25 & 4.9 & 9.8 & 5.3 & 0.54 & 3.7 \\
\hline December & 19 & 3.9 & 7.6 & 3.9 & 0.51 & 2.9 \\
\hline January & 17 & 3.2 & 6.6 & 3.6 & 0.55 & 2.5 \\
\hline February & 14 & 2.7 & 5.8 & 3.0 & 0.52 & 2.1 \\
\hline March & 8.1 & 2.6 & 4.4 & 2.6 & 0.36 & 1.7 \\
\hline Apr 11 & 15 & 2.4 & 6.1 & 3.7 & 0.61 & 2.3 \\
\hline May & 53 & 17 & 36 & 10 & 0.29 & 13.5 \\
\hline June & 117 & 49 & 83 & 22 & 0.27 & 31.5 \\
\hline July & 75 & 31 & 53 & 16 & 0.31 & 20.0 \\
\hline August & 34 & 15 & 23 & 6.4 & 0.27 & 8. 9 \\
\hline September & 25 & 11 & 16 & 4.9 & 0.30 & 6.2 \\
\hline Annual & 33 & 17 & 22 & 5.1 & 0.23 & 100 \\
\hline
\end{tabular}

Magnitude and frequency of instantaneous peak flow, based on perlod of record 1967-73, 1980-84

Discharge, in $\mathrm{ft}^{3 / \mathrm{s}}$, for indlcated recurrence interval, in years, and exceedance probablitty, in percent

\begin{tabular}{cccccc}
2 & 5 & 10 & 25 & 50 & 100 \\
50 & 20 & 10 & 41 & 21 & 11 \\
\hline 182 & 228 & 256 & 290 & 314 & 338
\end{tabular}

Duration table of dally mean flow for perlod of record 1967-73, 1980-84
Magnltude and frequency of annual low flow, based on pertod of record 1968-73, 1981-84

\begin{tabular}{|c|c|c|c|c|c|c|}
\hline \multirow{3}{*}{$\begin{array}{l}\text { Perlod } \\
\text { icon- } \\
\text { secu- } \\
\text { tive }\end{array}$} & \multicolumn{6}{|c|}{$\begin{array}{l}\text { Dlscharge, in ftos, for indlcated } \\
\text { recurrence interval, in years, and } \\
\text { nonexceedance probability, in percent }\end{array}$} \\
\hline & 7 & 5 & & & 500 & 1008 \\
\hline & 50 & $\begin{array}{c}5 \\
204\end{array}$ & $\begin{array}{l}10 \\
108\end{array}$ & 51 & 21 & 100 \\
\hline & & & & & & \\
\hline 1 & 2.7 & 2.0 & 1.6 & 1.4 & 1.1 & 0.99 \\
\hline 3 & 2.7 & 2.0 & 1.6 & 1.4 & 1.1 & 0.99 \\
\hline 7 & 2.8 & 2.0 & 1.7 & 1.4 & 1.2 & 0.99 \\
\hline 14 & 3.0 & 2,2 & 1.9 & 1.6 & 1.3 & 1.2 \\
\hline 30 & 3.7 & 3.0 & 2.6 & 2.3 & 2.0 & 1.9 \\
\hline 60 & 4.7 & 3.8 & 3.4 & 3.2 & 3.1 & 3.0 \\
\hline 90 & 5.0 & 4.0 & 3.7 & 3.6 & 3.5 & 3,4 \\
\hline 120 & 5.4 & 4.4 & 4.1 & 3.9 & 3.8 & 3.8 \\
\hline 183 & 7.0 & 5.7 & 5.4 & 5.3 & 5.1 & 5.1 \\
\hline
\end{tabular}



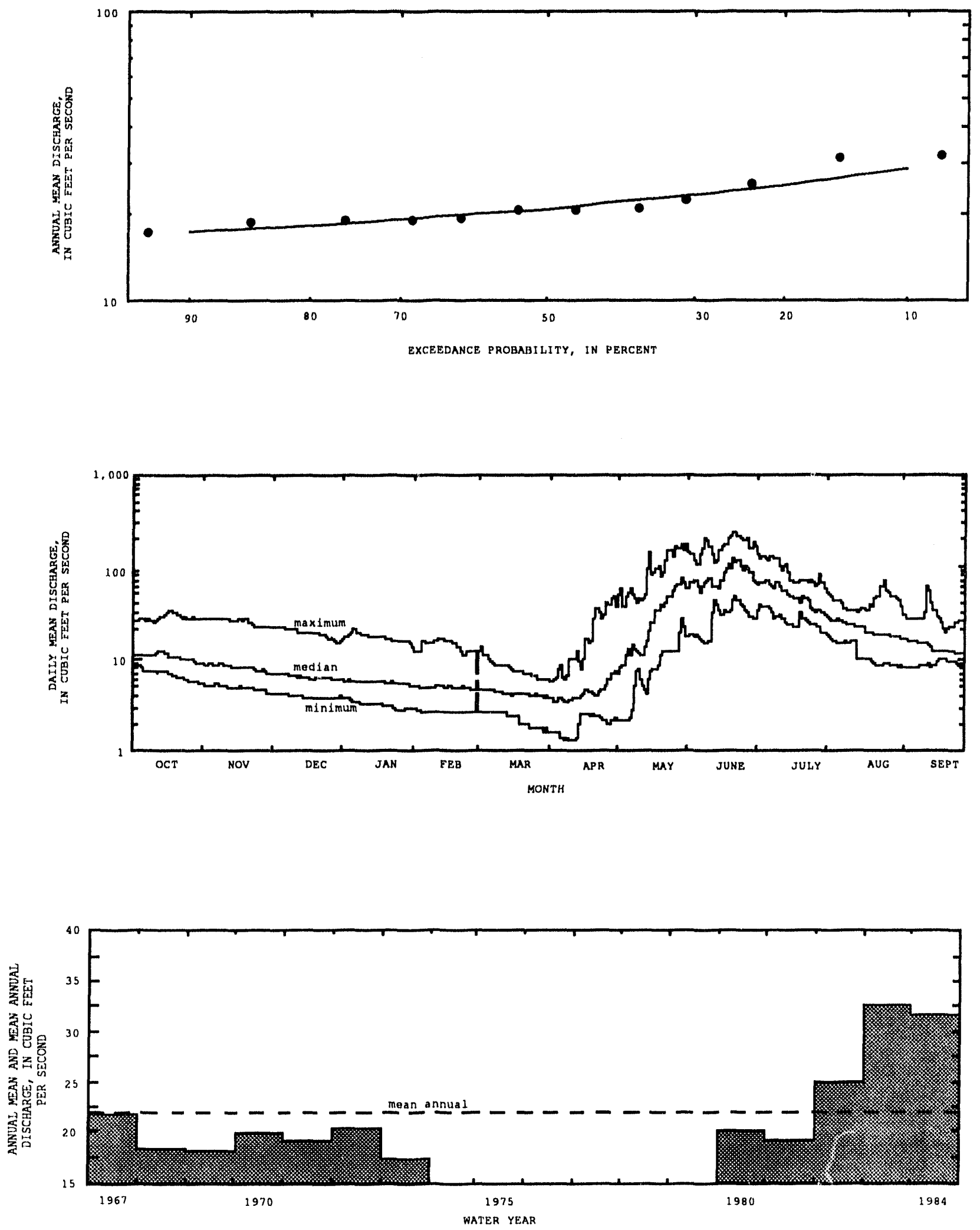


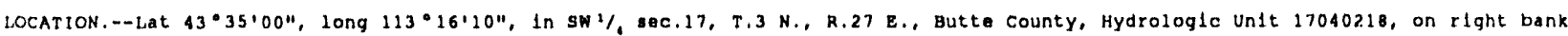
$0.4 \mathrm{ml}$ downatroam from slough entering from left bank, and $4 \mathrm{ml}$ southeast of Arco.

DRAINAGE AREA, $--1,410 \mathrm{ml}$ \%, approximately.

PERIOD OF RECORD.--Auguat 1946 to September 1980, March to September 1981 , May 1982 to september 1990.

GAGE. - Water-stage recorder. Elevation of gage 1. $5,240 \mathrm{ft}$ above sea level, by barometer. Prior to oct, 14 , 1952 , at alte 800 ft upatream at datum $3.08 \mathrm{ft}$ higher.

REMARKS.--Flow regulated by Mackay Reservolr. Station lo below all large diverglons for frrlgatlon in Blg lost Rlver Valley. About 57,500 acres or land irrlgated by dlversions from river and tributarles and by ground-water withdrawals above gtatlon. About 10,200 acres irrigated by aubirrigation above Mackay Reservolr.

EXTREMES FOR PERIOD OF RECORD,--Maximum discharge, $1,890 \mathrm{ft} 1 / \mathrm{s}$ July 5,1967 , gage holght, $7.68 \mathrm{ft}$; no $\mathrm{flow}$ on many days.

Summary of monthly and annual discharges, 1947-61, 1967-80, 1983-90

\begin{tabular}{|c|c|c|c|c|c|c|}
\hline Month & $\begin{array}{l}\text { Maximum } \\
(\mathrm{ft} / \mathrm{s})\end{array}$ & $\begin{array}{l}\text { Minlmum } \\
\left(f t^{3} / s\right)\end{array}$ & $\begin{array}{l}\text { Mean } \\
\left(\mathrm{ft}^{2} / \mathrm{s}\right)\end{array}$ & $\begin{array}{c}\text { stan- } \\
\text { dard } \\
\text { devia- } \\
\text { tton } \\
\left(\mathrm{ft}^{3} / \mathrm{a}\right)\end{array}$ & $\begin{array}{l}\text { Coeffi- } \\
\text { clent of } \\
\text { vart- } \\
\text { ation }\end{array}$ & $\begin{array}{l}\text { Per- } \\
\text { centage } \\
\text { of } \\
\text { annual } \\
\text { runoff }\end{array}$ \\
\hline October & 371 & 0.00 & 97 & 100 & 1.0 & 7.4 \\
\hline November & 759 & 0.00 & 102 & 137 & 1.3 & 7.7 \\
\hline December & 614 & 0.00 & OB & 111 & 1.3 & 6.7 \\
\hline January & 347 & 0.00 & 70 & 73 & 1.0 & 5.3 \\
\hline February & 314 & 0.00 & 72 & 69 & 0.96 & 5.5 \\
\hline March & 390 & 0.00 & 94 & 98 & 3.0 & 7.2 \\
\hline Apr 11 & 653 & 0.00 & 112 & 155 & 1.4 & B. 5 \\
\hline May & 841 & 0.00 & 140 & 212 & 1.5 & 10.6 \\
\hline June & 1,120 & 0.00 & 246 & 332 & 1.3 & 18.8 \\
\hline July & 918 & 0.00 & 145 & 243 & 1.7 & 11.0 \\
\hline August & 502 & 0.00 & 60 & 95 & 1.6 & 4.6 \\
\hline September & 395 & 0.00 & 88 & 105 & 1.2 & 6.7 \\
\hline Annual & 546 & 0.00 & 109 & 118 & 1.1 & 100 \\
\hline
\end{tabular}

Magnitude and frequency of instantaneoug peak flow, based on perlod of record 1947-61, 1967-80, $1983-90$

Dlscharge, in $f t^{3 / s}$, for Indicated recurrence Interval. in years, and exceedance probablitty, in percent

$\begin{array}{cccccc}2 & 5 & 10 & 25 & 50 & 100 \\ 501 & 201 & 101 & 41 & 21 & 14 \\ 420 & 1.100 & 1,530 & 2,350 & 3,010 & 3,700\end{array}$

Magnitude and frequency of annual low flow, based on perlod of record 1948-61, 1968-80,1984-91

\begin{tabular}{|c|c|c|c|c|c|c|}
\hline \multirow{3}{*}{$\begin{array}{l}\text { Parlod } \\
\text { (con- } \\
\text { secu- } \\
\text { tive } \\
\text { daya) }\end{array}$} & \multicolumn{6}{|c|}{$\begin{array}{l}\text { Discharge, In } \mathrm{ft} / \mathrm{s} \text {, for Indicated } \\
\text { recurrence interval, in years, and } \\
\text { nonexcoudance probablitty, in percent }\end{array}$} \\
\hline & 2 & 5 & 10 & 20 & 50 & 100 \\
\hline & 501 & 201 & 101 & 51 & 21 & 11 \\
\hline 1 & 6.6 & 0.00 & 0.00 & 0.00 & 0.00 & 0.00 \\
\hline 3 & 7.1 & 0.1 & 0.00 & 0.00 & 0.00 & 0.00 \\
\hline 7 & 8.2 & 0.2 & 0.00 & 0.00 & 0.00 & 0.00 \\
\hline 14 & 9.9 & 1.1 & 0.00 & 0.00 & 0.00 & 0.00 \\
\hline 30 & 14 & 2.3 & 0.00 & 0.00 & 0.00 & 0.00 \\
\hline 60 & 17 & 4.4 & 0.00 & 0.00 & 0.00 & 0.00 \\
\hline 90 & 26 & 5.9 & 0.00 & 0.00 & 0.00 & 0.00 \\
\hline 120 & 29 & 7.0 & 0.00 & 0.00 & 0.00 & 0.00 \\
\hline 183 & 58 & 8.4 & 0.00 & 0.00 & 0.00 & 0.00 \\
\hline
\end{tabular}

Magn t tude and frequency of annual high flow, based on perlod of record 1947-61,1967-80,1983-90

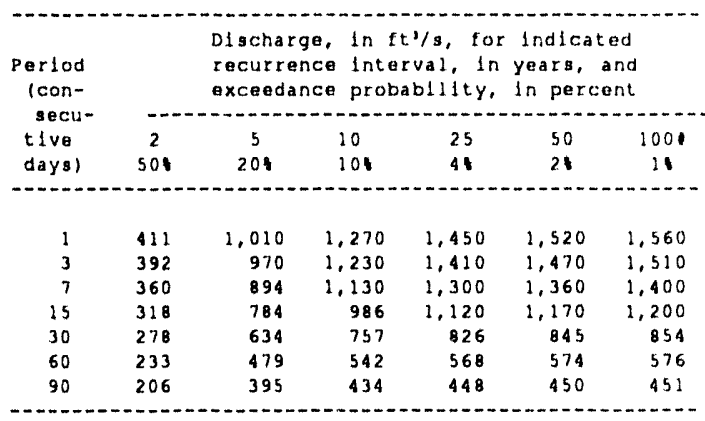

Duration table of dally mean flow for perlod of record 1947-61, 1967-80, 1983-90

Discharge, in $f t, 3$, which was excoeded for indlcated percentage of tine

\begin{tabular}{|c|c|c|c|c|c|c|c|c|c|c|c|c|c|c|c|c|}
\hline 11 & 51 & 104 & 151 & 201 & 304 & 401 & 504 & 601 & 701 & 804 & 901 & 951 & 981 & 991 & 99.51 & 99.91 \\
\hline 993 & 456 & 285 & 192 & 144 & 108 & 72 & 43 & 28 & 17 & 8.3 & 0.10 & 0.00 & 0.00 & 0.00 & 0.00 & 0.00 \\
\hline
\end{tabular}

- Length of record used in calculation may yleld unreliable values for this column. 

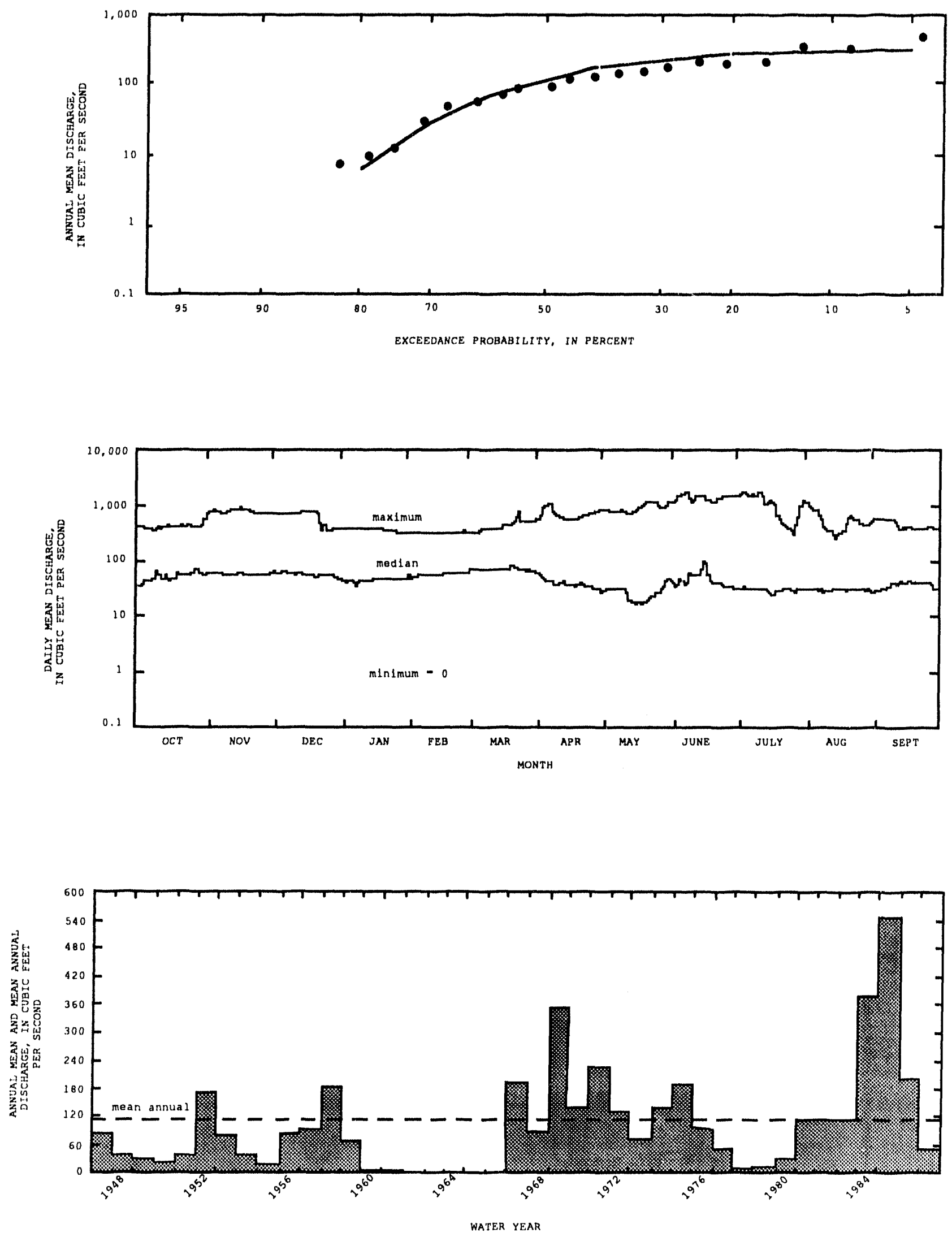
MUD LAKE-LOST RIVER BASIN

13132513 INEL DIVERSION AT HEAD NEAR ARCO, ID

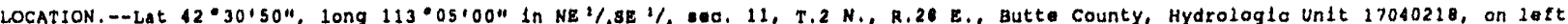
bank $0.05 \mathrm{ml}$ south of head of INEL diversion, $0.4 \mathrm{ml}$ north of Intersection of gravel road from HIghway $20-26$ and road on top of dike, and $13.2 \mathrm{ml}$ southwest of Arco.

PERIOD OF RECORD. - -1965-68 (dlscharge measurement only), July 1984 to september 1990.

GAGE.- Water-atage recorder and concrete control. Datum of gage lo $5,000.00$ it above sea level (levels by usGs).

REMARKS,--Flow is regulated by Mackay Reservolr and Is diverted from the Blg loat River for flood control at INEL facllitles. Graph of maxlmum-medlan-minimum discharge is based on entire perlod of record.

EXTREMES FOR PERIOU OF RECORD, --Maximum dally dlacharge, 1,290 ft/a June 9, 1986; no flow on many days.

summary of monthly and annual discharges, 1985-90

\begin{tabular}{|c|c|c|c|c|c|c|}
\hline Month & $\begin{array}{l}\text { Maximum } \\
(2 t: / a)\end{array}$ & $\begin{array}{l}\text { Mindmum } \\
\left(f t^{3} / s\right)\end{array}$ & $\begin{array}{r}\text { Mean } \\
\left(f t^{3} / a\right)\end{array}$ & $\begin{array}{l}\text { stan- } \\
\text { dard } \\
\text { devia- } \\
\text { tlon } \\
\left(\mathrm{ft}^{2} / \mathrm{a}\right)\end{array}$ & $\begin{array}{l}\text { Coeff } 1- \\
\text { clent of } \\
\text { var1- } \\
\text { atton }\end{array}$ & $\begin{array}{l}\text { Per- } \\
\text { centage } \\
\text { of } \\
\text { annual } \\
\text { xunoff }\end{array}$ \\
\hline October & 255 & 0.00 & 43 & 104 & 2.4 & 13.1 \\
\hline November & 194 & 0.00 & 40 & 78 & 2.0 & 12.2 \\
\hline December & 199 & 0.00 & 45 & 81 & 1.8 & 13.7 \\
\hline January & 138 & 0.00 & 31 & 56 & 1.8 & 9.3 \\
\hline February & 46 & 0.00 & 7.9 & 19 & 2.4 & 2.4 \\
\hline March & 110 & 0.00 & 24 & 44 & 1.8 & 7.4 \\
\hline Apr11 & 206 & 0.00 & 39 & 83 & 2.1 & 11.8 \\
\hline May & 63 & 0.00 & 11 & 25 & 2.4 & 3.3 \\
\hline June & 482 & 0.00 & 80 & 197 & 2.4 & 24,6 \\
\hline July & 0.00 & 0.00 & 0.00 & 0.00 & & 0.0 \\
\hline Auguat & 0.00 & 0.00 & 0.00 & 0.00 & & 0.0 \\
\hline September & 29 & 0.00 & 7.1 & 12 & 1.7 & 2.2 \\
\hline Annual & 94 & 0.00 & 27 & 38 & 1.4 & 100 \\
\hline
\end{tabular}

Duration table of dally mean flow for perlod of record $1985-90$

Discharge, in $f t 3 / s$, which was exceeded for Indlcated percentage of time

\begin{tabular}{|c|c|c|c|c|c|c|c|c|c|c|c|c|c|c|c|c|}
\hline 11 & 54 & 101 & 154 & 201 & 301 & 404 & 501 & 604 & 701 & 801 & 901 & 951 & 984 & 991 & 99.51 & 99.98 \\
\hline 350 & 199 & 76 & 42 & 1.3 & 0.03 & 0.03 & 0.02 & 0.02 & 0.01 & 0.01 & 0.00 & 0.00 & 0.00 & 0.00 & 0.00 & 0.00 \\
\hline
\end{tabular}

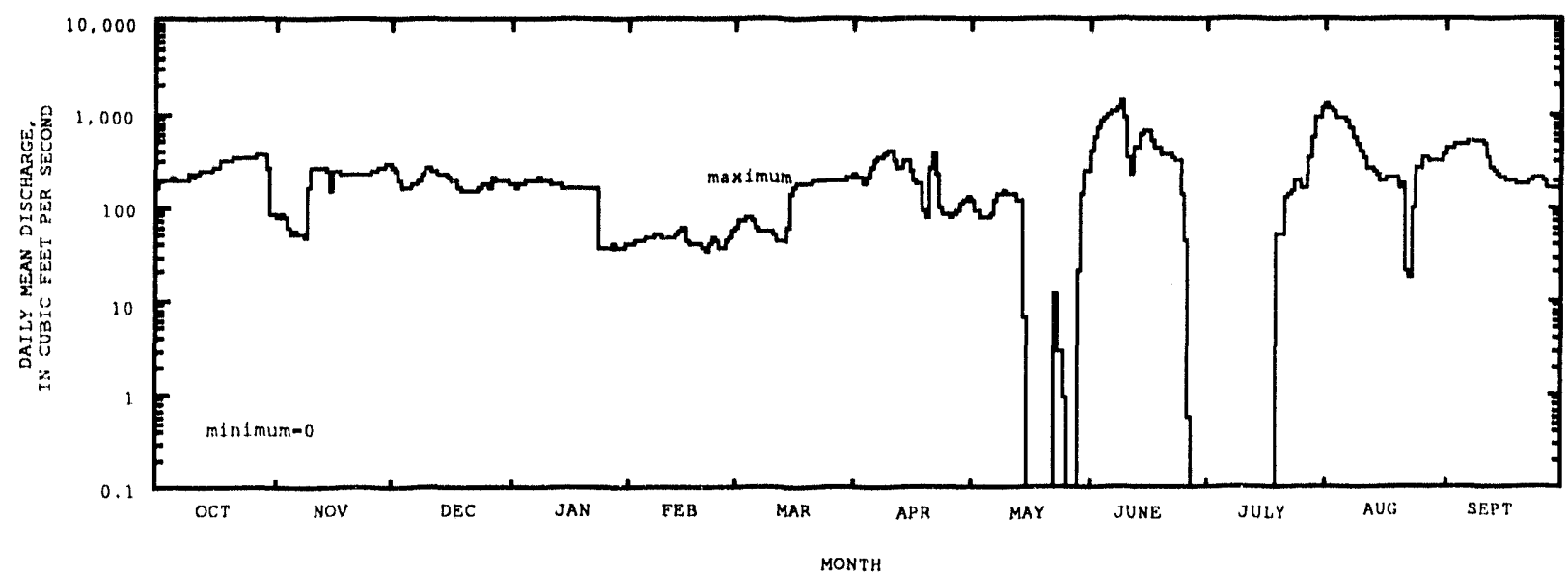


MUD LAKE-LOST RIVER BASIN

13132515 INEL DIVERSION AT OUTLET OF SPREADING AREA A NEAR ARCO, ID

LOCATION, --Lat $43^{\circ} 29145^{\prime \prime}$, long $113^{\circ} 04^{1} 13^{\prime \prime}$, In NE $1 /$ SW $1 /$ sec.13, T.2 N., R.28 E., Butte County, Hydrologlc Unit 17040218 , on left

bank $1.4 \mathrm{ml}$ south of head of INEL diveralon, $0.05 \mathrm{ml}$ south of outlet of spreading area A, and $14.5 \mathrm{ml}$ south of Arco.

PERIOD OF RECORD, $--1965-68$ (d1scharge measurements oniy); June 1984 to september 1990.

GAGE.-Water-stage recorder. Datum of gage 1 s $5,000.00 \mathrm{ft}$ above sea level (lovels by USGS).

REMARKS. - Flow 1 s requlated by Mackay Reservole and is diverted from the big lost Rlver tor flood control at INEL facilitieg. Graph of maximum-median-minlmum discharge is based on entire period of record.

EXTREMES FOR PERIOD OF RECORD,--Maximum dafly discharge, $989 \mathrm{ft} / \mathrm{g}$ June 9,1986 ; no flow on many days.

Summary of monthly and annual dischargea, $1985-90$

\begin{tabular}{|c|c|c|c|c|c|c|}
\hline Month & $\begin{array}{l}\text { Maxlmum } \\
(\mathrm{ft}, / \mathrm{s})\end{array}$ & $\begin{array}{l}\text { Minlmum } \\
\left(\mathrm{ft}^{3} / \mathrm{s}\right)\end{array}$ & $\begin{array}{r}\text { Mean } \\
\left(\mathrm{ft}^{3} / \mathrm{s}\right)\end{array}$ & $\begin{array}{c}\text { stan- } \\
\text { dard } \\
\text { devia- } \\
\text { tion } \\
\left(\mathrm{ft}^{2} / \mathrm{s}\right)\end{array}$ & $\begin{array}{l}\text { Coeffl- } \\
\text { clent of } \\
\text { vart- } \\
\text { ation }\end{array}$ & $\begin{array}{l}\text { Per- } \\
\text { centage } \\
\text { of } \\
\text { annual } \\
\text { runof } f\end{array}$ \\
\hline october & 220 & 0.00 & 37 & 90 & 2.4 & 14.8 \\
\hline November & 174 & 0.00 & 32 & 70 & 2.2 & 12.7 \\
\hline December & 180 & 0.00 & 36 & 72 & 2.0 & 14.5 \\
\hline January & 129 & 0.00 & 23 & 52 & 2.2 & 91 \\
\hline Fobruary & 4.1 & 0.00 & 0.69 & 1.7 & 2.4 & 0.3 \\
\hline March & 74 & 0.00 & 15 & 30 & 1.9 & 6.1 \\
\hline Apr 11 & 178 & 0.00 & 31 & 72 & 2.3 & 12,4 \\
\hline May & 34 & 0.00 & 5.8 & 14 & 2.4 & 2.3 \\
\hline June & 403 & 0.00 & 67 & 165 & 2.4 & 27.0 \\
\hline July & 0.00 & 0.00 & 0.00 & 0.00 & & 0.0 \\
\hline Auguat & 0.00 & 0.00 & 0.00 & 0.00 & & 0.0 \\
\hline September & 5.5 & 0.00 & 1.1 & 2.2 & 1.9 & 0.5 \\
\hline Annual & 80 & 0.00 & $2 \mathrm{i}$ & 33 & 1.6 & 100 \\
\hline
\end{tabular}

Duration table of dally mean flow for perlod of record 1985-90

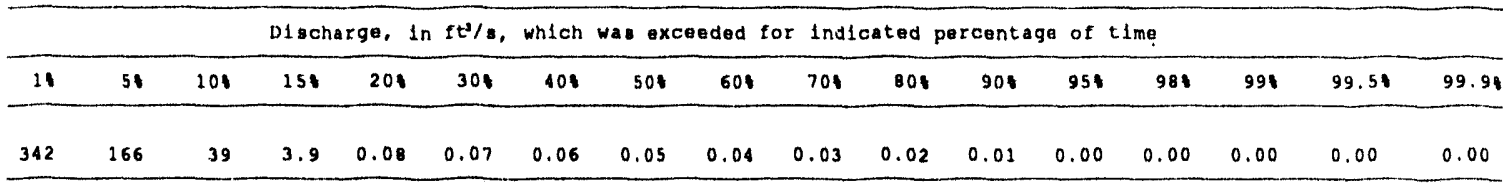

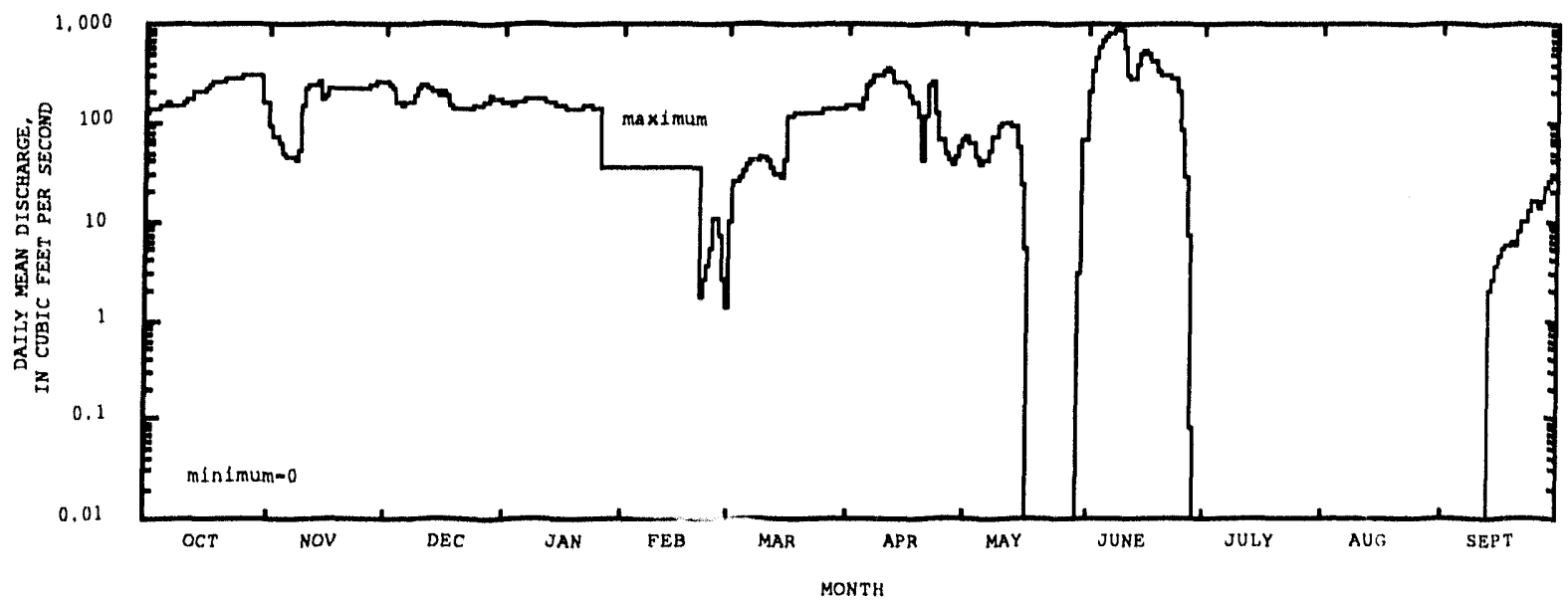


13132520 BIG LOST RIVER BELON INEL DIVERSION NEAR ARCO, ID

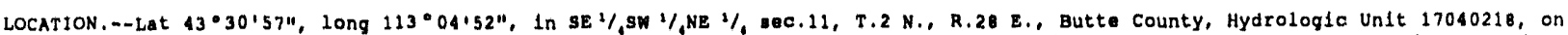
rlght bank $0.2 \mathrm{ml}$ north of the head of the $\mathrm{INEL}$ diversion, $4.5 \mathrm{ml}$ south of state H1ghway $20-26$ bridge over the Blg Lost River, and $13,2 \mathrm{mi}$ southeast of Arco.

PERIOD OF RECORD,--1965-68 (dlecharge messurements only)/ June 1984 to september 1990.

GAGE.--Water-stage recorder and concrete control. Datum of gage 1s 5,000.00 ft above sea level (levels by usgs).

REMARKs.-Flow regulated by Mackay Reservolr and INEL diversion. Station 1s below all diversions for 1rrigation in the Blg Lost River valley and $1 \mathrm{~g}$ below the INEL diversion for flood control. Graph of maximum-median-minimum discharge is based on entire period of record.

EXTREMES FOR PERIOD OF RECORD,--Maximum dally discharge, $460 \mathrm{ft} / \mathrm{s}$ June 10,1986 , no flow on many days.

Summary of monthly and annual flows, 1985-90

\begin{tabular}{|c|c|c|c|c|c|c|}
\hline Month & $\begin{array}{l}\text { Maximum } \\
\text { (ft } 1 / 8)\end{array}$ & $\begin{array}{l}\text { Mindmum } \\
\left(\mathrm{ft}^{1} / \mathrm{s}\right)\end{array}$ & $\begin{array}{r}\text { Mean } \\
\left(\mathrm{ft}^{\prime} / \mathrm{s}\right)\end{array}$ & $\begin{array}{c}\text { stan- } \\
\text { dard } \\
\text { devia- } \\
\text { tlon } \\
\left(\mathrm{ft}^{3} / \mathrm{s}\right)\end{array}$ & $\begin{array}{l}\text { Coeffi- } \\
\text { clent of } \\
\text { vart- } \\
\text { atton }\end{array}$ & $\begin{array}{l}\text { Per- } \\
\text { centage } \\
\text { of } \\
\text { annual } \\
\text { runoff }\end{array}$ \\
\hline October & 193 & 0.00 & 73 & 84 & 1.2 & 16.8 \\
\hline November & 166 & 0.00 & 37 & 65 & 1.7 & 0.5 \\
\hline December & 45 & 0.00 & 9.2 & 18 & 1.9 & 2.1 \\
\hline January & 21 & 0.00 & 4.7 & 0.4 & 1.8 & 1.1 \\
\hline Fobruary & 23 & 0.00 & 8.0 & 9.6 & 1.2 & 1.8 \\
\hline March & 59 & 0.00 & 15 & 24 & 1.6 & 3.5 \\
\hline Apr11 & 183 & 0.00 & 58 & 80 & 1.4 & 13.3 \\
\hline May & 281 & 0.00 & 68 & 115 & 1.7 & 15.6 \\
\hline June & 392 & 0.00 & 67 & 159 & 2.4 & 15.3 \\
\hline July & 58 & 0.00 & 11 & 23 & 2.2 & 2.4 \\
\hline August & 38 & 0.00 & 6.5 & 15 & 2.3 & 1.5 \\
\hline September & 270 & 0.00 & 79 & 124 & 1.6 & 18.1 \\
\hline Annua 1 & 127 & 0.00 & 36 & 52 & 1.4 & 100 \\
\hline
\end{tabular}

Duration table of dally mean flow for perlod of record 1985-90

Discharge, in $\mathrm{ft}^{3} / \mathrm{a}$, which was exceeded for indicated percentage of time

\begin{tabular}{lllllllllllllllllll}
\hline 1 & 51 & 104 & 154 & 204 & 304 & 401 & 504 & 604 & 70 & 80 & 904 & 95 & 984 & 994 & 99.54 & 99.98 \\
398 & 251 & 130 & 71 & 32 & 8.5 & 0.04 & 0.03 & 0.03 & 0.02 & 0.01 & 0.01 & 0.00 & 0.00 & 0.00 & 0.00 & 0.00 \\
\hline
\end{tabular}

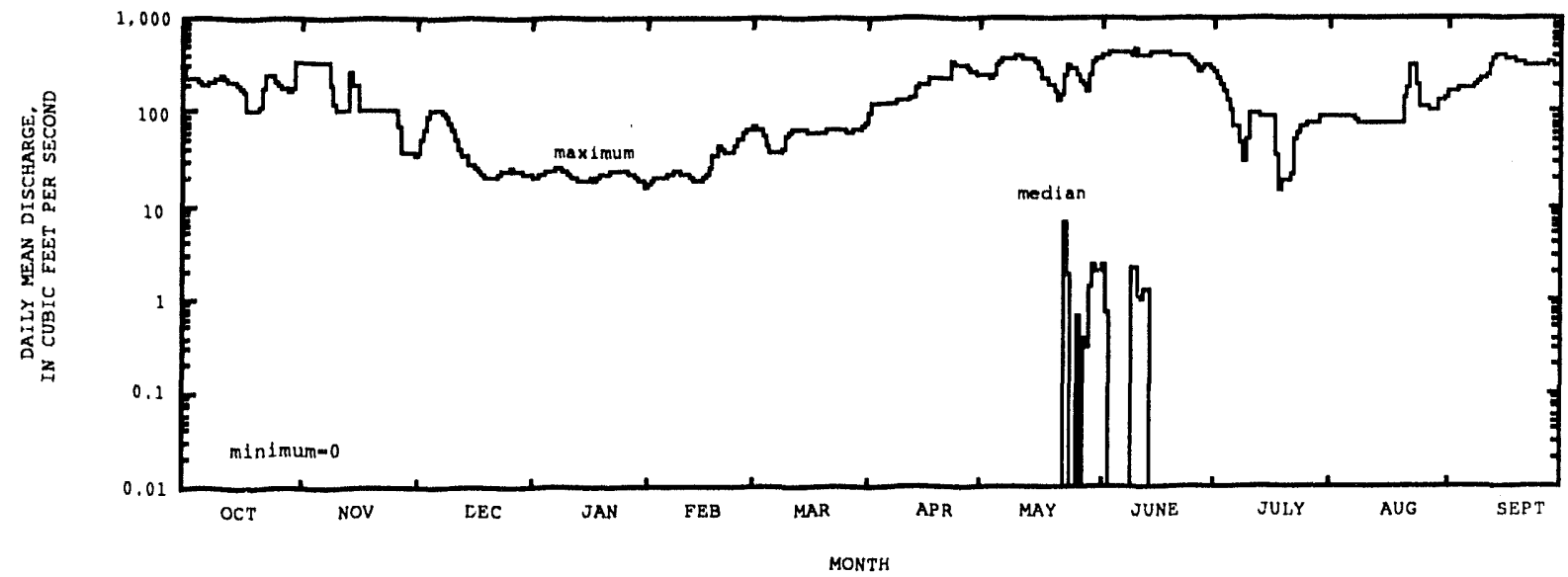


13132535 BIG LOST RIVER AT LINCOUN BOULEVARD BRIDGE NEAR ATOMIC CITY, ID

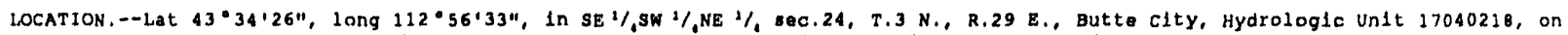
left bank $2.6 \mathrm{ml}$ north of Lincoln Boulevard-Portland Avenue intersection, and $18.5 \mathrm{ml}$ southeast of Arco.

PERIOD OF RECORD,--1951-53, 1957, 1965-60 (d1scharge measurements only): July 1984 to september 1990.

GAGE.--Water-gtage recorder. Datum of gage 1s 4,900.00 ft above sea level (levelg by USGS).

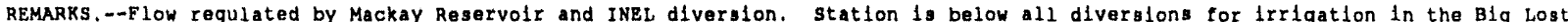
River Valley and is below the INEL diversion for flood control. Graph of maximum-median-minimum discharge $1 \mathrm{~s}$ based on entire pertod of record.

EXTREMES FOR PERIOD OF RECORD.--Maximum discharge, $393 \mathrm{ft} / \mathrm{s}$ June 10, 1986, gage height, $15.91 \mathrm{ft}$ : no flow on many days.

Summary of monthly and annual discherges, 1985-90

\begin{tabular}{|c|c|c|c|c|c|c|}
\hline Month & $\begin{array}{l}\text { Maximum } \\
(\mathrm{ft} 1 / \mathrm{s})\end{array}$ & $\begin{array}{l}\text { Minimum } \\
\left(\mathrm{ft}^{1 / 8} / \mathrm{s}\right)\end{array}$ & $\begin{array}{l}\text { Mean } \\
\left(\mathrm{ft}^{\prime} / \mathrm{s}\right)\end{array}$ & $\begin{array}{c}\text { stan- } \\
\text { dard } \\
\text { devia- } \\
\text { tion } \\
\left(\mathrm{ft}^{2} / \mathrm{s}\right)\end{array}$ & $\begin{array}{l}\text { Coeffi- } \\
\text { clent of } \\
\text { vart- } \\
\text { ation }\end{array}$ & $\begin{array}{l}\text { Per- } \\
\text { centage } \\
\text { of } \\
\text { annual } \\
\text { runoff }\end{array}$ \\
\hline October & 174 & 0.00 & 68 & 78 & 1.2 & 19.5 \\
\hline November & 146 & 0.00 & 31 & 57 & 1.8 & 9.0 \\
\hline December & 35 & 0.00 & 5.8 & 14 & 2.4 & 1.7 \\
\hline January & 0.05 & 0.00 & 0.01 & 0.02 & 2.4 & 0.0 \\
\hline February & 1.7 & 0.00 & 0.29 & 0.70 & 2.4 & 0.1 \\
\hline March & 8. 7 & 0.00 & 2.9 & 4.5 & 1.5 & 0.8 \\
\hline Apr11 & 141 & 0.00 & 45 & 67 & 1.5 & 13.0 \\
\hline May & 241 & 0.00 & 58 & 99 & 1.7 & 16.6 \\
\hline June & 341 & 0.00 & 57 & 139 & 2.4 & 16.4 \\
\hline July & 17 & 0.00 & 7.9 & 19 & 2.4 & 2.3 \\
\hline August & 17 & 0.00 & 2.8 & 6.7 & 2.4 & 0.8 \\
\hline September & 248 & 0.00 & 69 & 110 & 1.6 & 19.8 \\
\hline Annua I & 103 & 0.00 & 29 & 42 & $\therefore .5$ & 100 \\
\hline
\end{tabular}

Duration table of dally mean flow for perlod of record 1985-90

\begin{tabular}{|c|c|c|c|c|c|c|c|c|c|c|c|c|c|c|c|c|}
\hline & & & D1sch & ge, 1 & $\mathrm{ft} / \mathrm{s}$, & wh1ch & was exc & eded $f$ & r Inds & sated & $r$ centa & le of & & & & \\
\hline $1 \%$ & 54 & 101 & $15 t$ & 201 & 301 & 404 & 501 & 604 & 701 & 801 & 901 & 95 & $98 t$ & 994 & 99.51 & 99.91 \\
\hline 343 & 233 & 111 & 50 & 6.8 & 0.18 & 0.16 & 0.13 & 0.10 & 0.08 & 0.05 & 0.03 & 0.01 & 0.00 & 0.00 & 0.00 & 0.00 \\
\hline
\end{tabular}

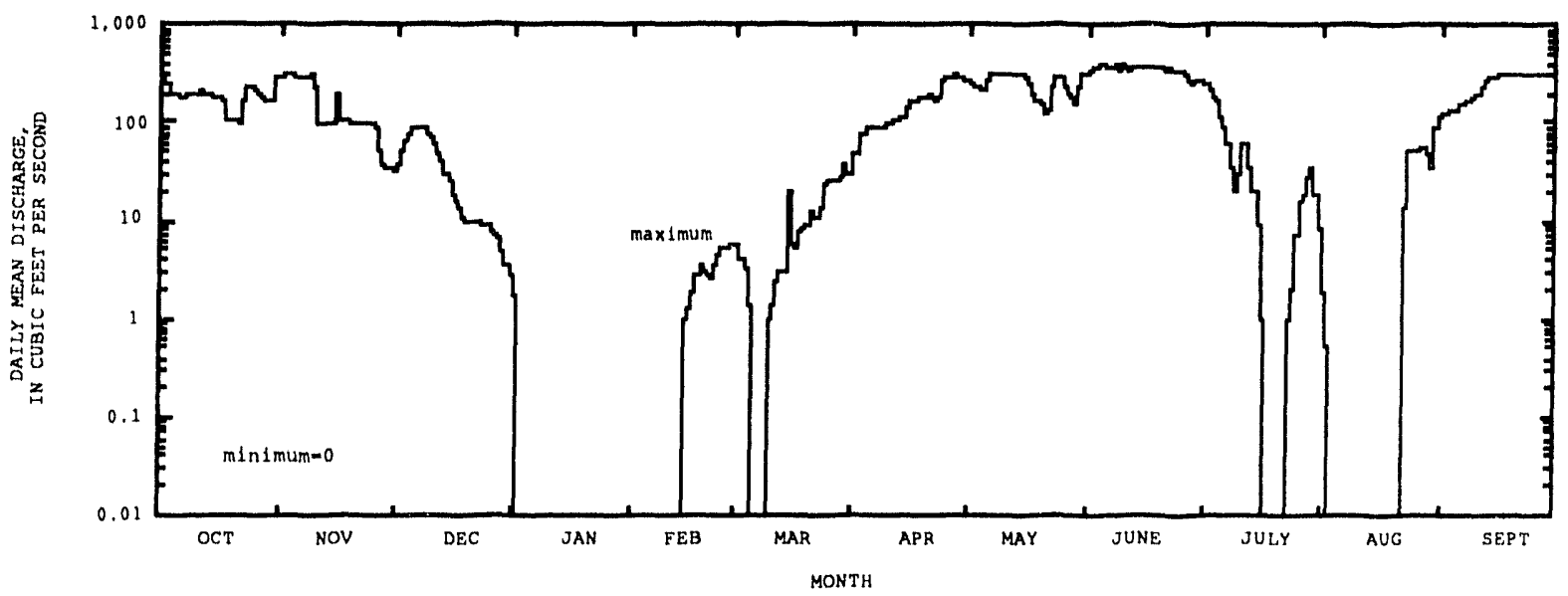



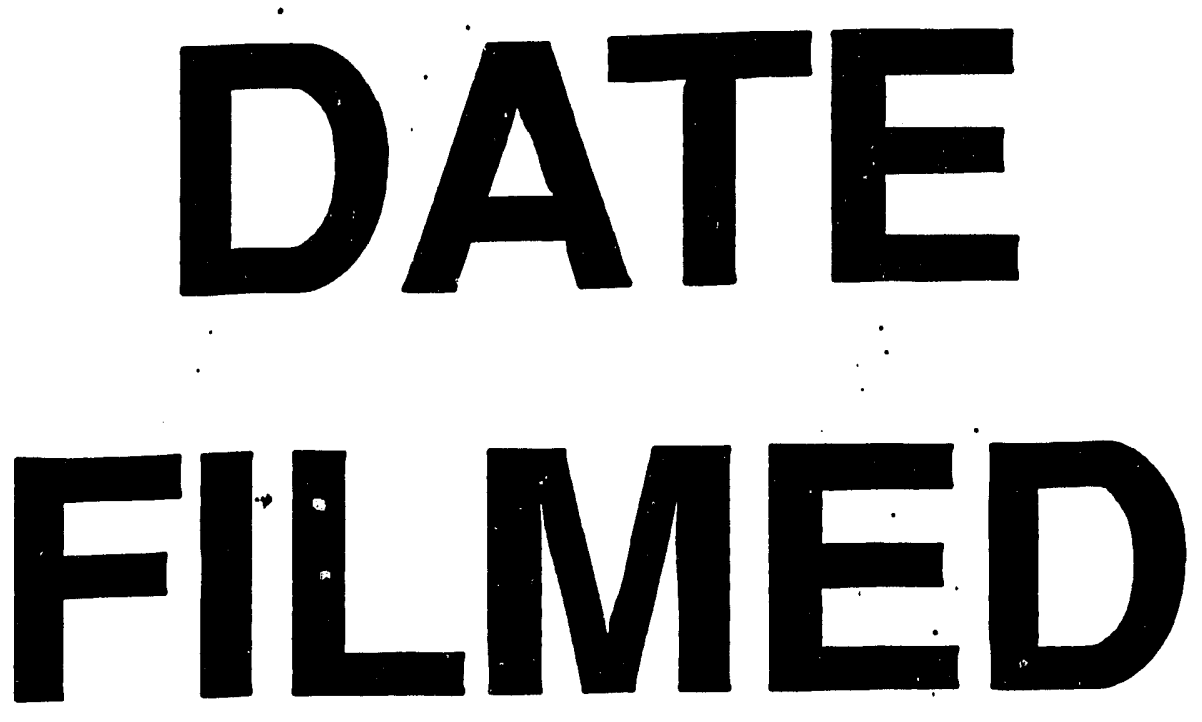

$1 / 6 / 94$
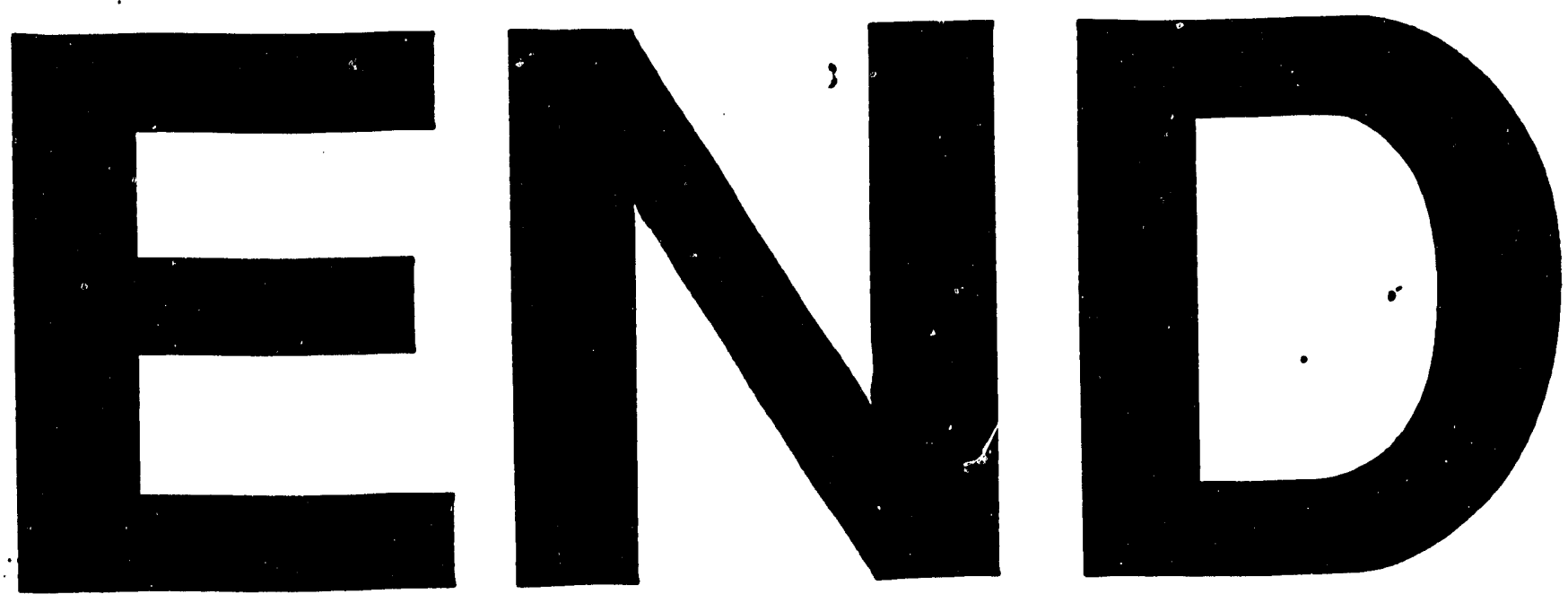
